\title{
A Colocalized Scheme for Three-Temperature Grey Diffusion Radiation Hydrodynamics
}

\author{
R. Chauvin ${ }^{1}$, S. Guisset ${ }^{1, *}$, B. Manach-Perennou ${ }^{1}$ and L. Martaud ${ }^{1}$ \\ ${ }^{1}$ CEA, DAM, DIF, F-91297 Arpajon, France.
}

Received 25 March 2021; Accepted (in revised version) 25 August 2021

\begin{abstract}
A positivity-preserving, conservative and entropic numerical scheme is presented for the three-temperature grey diffusion radiation hydrodynamics model. More precisely, the dissipation matrices of the colocalized semi-Lagrangian scheme are defined in order to enforce the entropy production on each species (electron or ion) proportionally to its mass as prescribed in [34]. A reformulation of the model is then considered to enable the derivation of a robust convex combination based scheme. This yields the positivity-preserving property at each sub-iteration of the algorithm while the total energy conservation is reached at convergence. Numerous pure hydrodynamics and radiation hydrodynamics test cases are carried out to assess the accuracy of the method. The question of the stability of the scheme is also addressed. It is observed that the present numerical method is particularly robust.
\end{abstract}

AMS subject classifications: 65M12, 35Q35, 82D10, 82A25

Key words: Colocalized Lagrangian scheme, radiation hydrodynamics, grey diffusion, discrete entropy production, plasma physics simulations.

\section{Introduction}

\section{Background}

Considering a non-equilibrium plasma, the relaxation process to reach the electronic and ionic temperature equalization occurs on time scales much shorter than the ones involved to reach quasi-neutral regimes or electron and ion Maxwellian equilibrium distribution functions [7]. This must be highlighted since in many practical applications, such as astrophysics or inertial confinement fusion, the characteristic times of interest can be of the same order of the temperature relaxation times. When this is the case, a two-temperature

*Corresponding author. Email addresses: sebastien.guisset@cea.fr (S. Guisset), remi . chauvin@cea.fr (R. Chauvin), bastien.manach-perennou@cea.fr (B. Manach-Perennou), ludovic.martaud@cea.fr (L. Martaud) 
hydrodynamics model is required [7]. In the presence of strong radiation fields, the modeling of photon transport is also mandatory. Various physical descriptions are available depending on the level of accuracy needed [22]. Here, we restrict ourselves to the study of a three-temperature grey diffusion radiation hydrodynamics model, which is only valid in the optically thick limit [22].

The numerical resolution of radiation hydrodynamics models has been heavily investigated over the years. If one requires a transport-type modeling, among other methods to simulate thermal radiation propagation is the Implicit Monte-Carlo method. Early investigations may be found in [12] where the Implicit Monte-Carlo method is used for a two-temperature (radiation and matter temperatures) model. See also [9] in which the Implicit Monte-Carlo method is extended to the study of three-temperature models. We also mention here that angular moments $(\mathrm{PN})$ and discrete ordinate $(\mathrm{SN})$ methods are also widely used in this context $[4,22]$. If the physical problem studied allows diffusiontype approximations, i.e if the photon mean free path is very small compared to the characteristic length of the problem (optically thick media) $[13,25]$, then standard NewtonRaphson or fix point strategy are also widely used to solve three-temperature models. Here it is assumed that the photon spectrum is Planckian and the radiation field is determined by many absorptions and re-emissions. In this case the radiation field rapidly becomes Planckian at a temperature not necessarily same as the material. We refer to [10] for numerical comparisons between several simulation codes for solving three-temperature models. It should also be mentioned that Jacobian-free Newton-Krylov methods enable the derivation of efficient algorithms for radiation diffusion equations [11,24]. Finally, notice that the time integration strategy of non-equilibrium radiation diffusion models has been investigated in [16] and references therein. We also mention here that purely diffusive model has a limited applicability and flux-limiting techniques are usually used [25]. The integration of limiting techniques to the numerical strategy presented in this document does not pose any issue and is not mentioned.

The numerical resolution of three-temperature models has become an active research field in recent years. In $[29,30]$ the numerical resolution of a three-temperature radiation hydrodynamics model on unstructured grids is presented. Concerning the numerical resolution of diffusion operators on (strongly) deformed meshed we refer to $[1,3,26]$ and the references therein. We also mention that very recent studies have been published, specifically dealing with the positive preserving (or maximum principle preserving) properties for three-temperature radiation diffusion equations [27,33].

In the present study, we do not consider pure Eulerian strategies. Indeed, even if large material deformation may be handled by Euler codes, their diffusive feature makes multi-material configurations difficult to handle. Of course, theses issues may be addressed with advanced material interface tracking techniques [28] inside mixed cells. However and more importantly, the discrete entropy production (per species) is almost never studied (or even mentioned) when working with pure Euler formalism. Gibb's relation cannot be easily combined with the evolution equations so that this key point is often neglected. Even if one could expect a global entropy production by the scheme, the 
species entropy production is generally unclear. On the contrary, Lagrangian codes are naturally less diffusive since the nodes follow the flow and therefore are naturally well suited for multi-material configurations as well as handling source terms. In addition as it will be seen in the present study, the discrete species entropy production may be carefully controlled to "mimic" species physical production. Of course, if large deformations occur, the appearance of strongly distorted cells makes Lagrangian approaches less robust. To bypass this difficulty the numerical solution may be projected on a regular mesh (Lagrangian plus remap strategy) considering an arbitrary Lagrangian Eulerian scheme (ALE) [14]. If the projection step is performed carefully, the overall numerical strategy leads to the Lagrangian advantages such as natural multi-physics couplings, sharp interfaces between materials or accuracy in addition to the robustness of the Eulerian-type approaches.

\section{Present approach and outline}

The present approach starts from the colocalized Lagrangian scheme presented in [20] which naturally ensures the geometric conservation law (GCL), and is extended to the three-temperature formalism. The authors are aware of staggered mesh formulation schemes in which the velocity are defined at the nodes and the other variables at the cell centers, working with the use of an artificial viscosity. It will be seen that despite the diffusive character of the scheme, since it is originally based on a standard (HLLC-type) Godunov solver, the numerical dissipation may be "tuned" while keeping the numerical stability and achieving a physical discrete species entropy production. In addition, since all variables are located at the cell centers the remap step used here is straightforward. In the present case, the solution is simply projected onto the initial (Cartesian) mesh. To extend the standard colocalized approach of [20] (in which a total energy evolution equation is used) to the three-temperature formalism, internal energy equations for electrons, ions and photons are considered. Similar ideas may be found in [6] in which a colocalized Lagrangian scheme is used for solving multiple internal energy equations, while keeping the total energy conservation property. However, the discrete entropy production issue, which is a key feature for the applications of interest (multi-material compressible flow for inertial confinement fusion), is never addressed. In the present case the dissipation matrices of the colocalized semi-Lagrangian scheme are defined in order to enforce entropy production on each species with respect to its mass fraction as prescribed in [34]. This point is explained in details in the present study.

In [2], in the case of a two-temperature (electron and ion) model the numerical strategy followed consists in computing the total energy first with a standard colocalized scheme [20] working with a total energy evolution equation. Then, considering an isotropic flow for electrons, the electron internal energy is easily computed and the ionic internal energy may be recovered. Finally, electron and ion conductivities and electron-ion coupling term are taken into account using a standard Newton procedure. The present approach is genuinely different since no operator-splitting strategy is required (except for the electron and ion conductivities) and electron, ion and radiation energies are com- 
puted simultaneously. The positiveness of the discrete temperatures may then be ensured.

We believe the originality of the present work comes from the following reasons. All the fundamental numerical properties, discrete entropy production (per species), discrete total energy conservation, discrete temperatures positiveness are proved for the full three-temperature radiation hydrodynamics model. These properties are often partially proved only for the hydrodynamics or the diffusion part. In addition, the large numerical tests carried out, demonstrate that the overall methodology proposed is particularly robust and is naturally well-suited for multi-physics coupling.

The document is organized as follows. Firstly, the three-temperature model is presented with its main properties. These properties will latter be enforced at the discrete level. In a second part, the derivation of the numerical schemes is carried out to enforce the discrete total energy conservation as well as the entropic character. More precisely, the dissipation matrices of the colocalized semi-Lagrangian scheme are defined in order to enforce the species entropy production on ions as prescribed in [34]. A reformulation of the model is then considered to enable the derivation of a robust convex-combination based scheme. This yields the positivity-preserving property at each sub-iteration of the algorithm while the total energy conservation is reached at convergence. Finally, numerous pure hydrodynamics and radiation hydrodynamics test cases are carried out to assess the accuracy of the method. The question of the stability of the scheme is also addressed. It is observed that the present numerical method is particularly robust.

\section{Three-temperature plasma model}

In this section, the physical model studied is presented with its main properties. In the next sections, these properties will then be enforced at the discrete level.

\subsection{Model and notations}

In order to model the evolution of ions, electrons and photons contained in a plasma, we consider the following three-temperature model written in updated Lagrangian formalism $[2,23]$

$$
\left\{\begin{array}{l}
\rho \frac{\mathrm{d}}{\mathrm{d} t}\left(\frac{1}{\rho}\right)-\nabla \cdot \boldsymbol{u}=0, \\
\rho \frac{\mathrm{d} \boldsymbol{u}}{\mathrm{d} t}+\nabla p=0, \quad p=p_{r}+p_{e}+p_{i}, \\
\rho \frac{\mathrm{d}}{\mathrm{d} t}\left(\frac{E_{r}}{\rho}\right)+p_{r} \nabla \cdot \boldsymbol{u}+\nabla \cdot \boldsymbol{F}_{r}=c \sigma_{P}\left(a T_{e}^{4}-E_{r}\right), \\
\rho \frac{\mathrm{d} \varepsilon_{e}}{\mathrm{~d} t}+p_{e} \nabla \cdot \boldsymbol{u}+\nabla \cdot \boldsymbol{F}_{e}=c \sigma_{P}\left(E_{r}-a T_{e}^{4}\right)+\kappa\left(T_{i}-T_{e}\right), \\
\rho \frac{\mathrm{d} \varepsilon_{i}}{\mathrm{~d} t}+p_{i} \nabla \cdot \boldsymbol{u}+\nabla \cdot \boldsymbol{F}_{i}=\kappa\left(T_{e}-T_{i}\right),
\end{array}\right.
$$


where $c$ and $a$ denote the speed of light and the radiation constant respectively. In addition, $\rho$ is the density of the fluid, $u$ denotes the speed of the fluid and $p$ is the total pressure obtained by summing the pressure of ions $p_{i}$, the pressure of electrons $p_{e}$ and the radiative pressure $p_{r} . E_{r}$ is the volumetric density of radiative energy, $\varepsilon_{e}$ and $\varepsilon_{i}$ denote the specific electronic and ionic energy densities. $\sigma_{P}$ is the Planck opacity which is a given non-linear function of $T_{e}$ and $T_{r}\left(E_{r}=a T_{r}^{4}\right)$. Let $\kappa$ be a positive relaxation coefficient of the electronic and ionic temperatures which depends on $T_{e}$ and $T_{i}$. The radiative flux $F_{r}$ is chosen according to the Rosseland diffusion approximation [23]

$$
\boldsymbol{F}_{r}=-\frac{c}{3 \sigma_{R}\left(T_{e}\right)} \nabla E_{r}
$$

where the Rosseland opacity $\sigma_{R}$ is a function of electronic temperature $T_{e}$. In the same way, the radiative pressure $p_{r}$ is related to the radiative energy $E_{r}$ according to the relation $[4,23]$

$$
p_{r}=E_{r} / 3 \text {. }
$$

Finally, a standard Spitzer-Harm model is chosen for electronic and ionic conductivities [31]

$$
\boldsymbol{F}_{\alpha}=K_{\alpha} T_{\alpha}^{5 / 2} \nabla T_{\alpha}, \quad \alpha \in\{e, i\} .
$$

In the present study, the electron and ion conduction terms are taken into account using standard operator splitting strategies. These terms are then removed from the numerical analysis which follows since they do not directly contribute in the scheme derivation and their discretization is straightforward. To conclude this section, we recall that by removing the diffusion and coupling terms in (1.1), the resulting system is hyperbolic with eigenvalues $\left(0,0,0,-c_{s}, c_{s}\right)$ where the sound speed, in the case of an ideal gas closure, is defined by

$$
c_{s}=\sqrt{\gamma\left(p_{e}+p_{i}\right) / \rho+p_{r} / \rho} .
$$

In the next section some properties of the model are presented.

\subsection{Main properties}

In this section the main model properties are briefly presented. In the following sections, they will be enforced at the discrete level.

\section{Energy conservation}

From the second equation of system (1.1) the kinetic energy equation reads

$$
\frac{\rho}{2} \frac{\mathrm{d}\|\boldsymbol{u}\|_{2}^{2}}{\mathrm{~d} t}+\nabla p \cdot \boldsymbol{u}=0
$$


Adding Eq. (1.5) with the three last equations of (1.1) gives the total energy conservation relation

$$
\rho \frac{\mathrm{d}}{\mathrm{d} t}\left(\frac{E_{r}}{\rho}+\varepsilon_{e}+\varepsilon_{i}+\frac{\|\boldsymbol{u}\|_{2}^{2}}{2}\right)+\nabla \cdot\left(p \boldsymbol{u}+\boldsymbol{F}_{r}\right)=0 .
$$

We recall here that the electron and ion conduction terms have been removed from the numerical analysis.

\section{Positiveness}

It is considered that the electronic, ionic and radiation temperatures remain positive at all times. Such a property is studied in [17].

\section{Total entropy production}

The total specific entropy of the system is dissipated. In order to show the total entropy production, one considers the total specific entropy of the system $\eta=\eta^{r}+\eta^{e}+\eta^{i}$, where $\eta^{\alpha}$ is the specific entropy of the species $\alpha$. For the electrons and ions, consider the following equation (Gibbs relation) which defines the specific entropy for each species

$$
T_{\alpha} \mathrm{d} \eta^{\alpha}=\mathrm{d} \varepsilon_{\alpha}+p_{\alpha} \mathrm{d}\left(\frac{1}{\rho}\right), \quad \forall \alpha \in\{e, i\} .
$$

Similarly, the photon entropy is defined with the following relation

$$
T_{r} \mathrm{~d} \eta^{r}=\mathrm{d}\left(\frac{E_{r}}{\rho}\right)+p_{r} \mathrm{~d}\left(\frac{1}{\rho}\right)
$$

From (1.7) and (1.8), for smooth flows it is easy to show that

$$
\left\{\begin{array}{l}
\rho T_{r} \frac{\mathrm{d} \eta^{r}}{\mathrm{~d} t}=\nabla \cdot \frac{c}{3 \sigma_{R}} \nabla E_{r}+c \sigma_{P}\left(a T_{e}^{4}-E_{r}\right), \\
\rho T_{e} \frac{\mathrm{d} \eta^{e}}{\mathrm{~d} t}=c \sigma_{P}\left(E_{r}-a T_{e}^{4}\right)+\kappa\left(T_{i}-T_{e}\right), \\
\rho T_{i} \frac{\mathrm{d} \eta^{i}}{\mathrm{~d} t}=\kappa\left(T_{e}-T_{i}\right) .
\end{array}\right.
$$

so that for regular flows, the total entropy production $\eta=\eta^{r}+\eta^{e}+\eta^{i}$ is due to radiation and collision terms

$$
\begin{aligned}
\rho \frac{\mathrm{d} \eta}{\mathrm{d} t}= & \rho \frac{\mathrm{d} \eta^{r}}{\mathrm{~d} t}+\rho \frac{\mathrm{d} \eta^{e}}{\mathrm{~d} t}+\rho \frac{\mathrm{d} \eta^{i}}{\mathrm{~d} t} \\
= & \frac{1}{T_{r}} \nabla \cdot\left(\frac{c}{3 \sigma_{R}} \nabla E_{r}\right)+c \sigma_{P}\left(\frac{a T_{e}^{4}-E_{r}}{T_{r}}\right)+c \sigma_{P}\left(\frac{E_{r}-a T_{e}^{4}}{T_{e}}\right) \\
& +\kappa\left(\frac{T_{i}-T_{e}}{T_{e}}\right)+\kappa\left(\frac{T_{e}-T_{i}}{T_{i}}\right),
\end{aligned}
$$




$$
\begin{aligned}
= & \nabla \cdot\left(\frac{c}{3 \sigma_{R} T_{r}} \nabla E_{r}\right)+\frac{4 a c T_{r}}{3 \sigma_{R}}\left\|\nabla T_{r}\right\|^{2}+\frac{a c \sigma_{P}}{T_{r} T_{e}}\left(T_{e}-T_{r}\right)\left(T_{e}^{4}-T_{r}^{4}\right) \\
& +\kappa \frac{\left(T_{e}-T_{i}\right)^{2}}{T_{e} T_{i}} .
\end{aligned}
$$

For discontinuous solutions (shock waves), the last equality becomes an inequality. The first term of the right hand side of the inequality is expressed in a conservative form while the others are always positive. Consequently, by neglecting possible contributions at the boundaries, the total entropy in the domain is dissipated.

\section{Species entropy production}

In addition to this entropy production result, it is well-known [34] that as a shock wave propagates into the plasma medium, kinetic energy is converted into internal energy so that species specific entropy is dissipated proportionally to the mass of the species considered. The total entropy production is then mainly due to the ions contribution. More precisely, the ionic entropy production is $m_{i} / m_{e}$ times higher than the electron entropy production, where $m_{i}$ and $m_{e}$ denote respectively the ion and electron mass. The numerical scheme derivation will also be performed in order to enforce this property at the discrete level. Notice that this choice is more a physical one than a numerical one. Another choice can be made leading to different results at convergence.

\section{Numerical discretizations}

In this section the numerical strategy is presented. The derivation is performed in order to enforce the total energy conservation as well as a correct species entropy production.

\subsection{Geometry and notations}

The notations used are similar to the ones introduced in [19]. Each cell is assigned a unique index $c$ and is denoted $\omega_{c}$ with a volume $v_{c}$. The cells are assumed to be polygonal so that they are defined by the set of their nodes $\mathcal{P}(c)$. The neighbor cells are collected in the set $\mathcal{N}(c)$. For a given node $p, \mathcal{C}(p)$ is the set of cells that contains $p$. We write $x_{p}$ and $u_{p}$ for its position and velocity. We denote $p^{+}$the node in $\mathcal{P}(c)$ which follows $p$ in counterclockwise order and $p^{-}$the previous node. Let $\boldsymbol{n}_{p c}^{+}$be the outward normal unit vector to $p \vec{p}^{+}$and consider $l_{p c}^{+}=\frac{1}{2}\left\|p \vec{p}^{+}\right\|$. Similarly, $n_{p c}^{-}$and $l_{p c}^{-}$are defined accordingly. Consequently, the corner outward normal unit vector $\boldsymbol{n}_{p c}$ is defined as follows

$$
\boldsymbol{n}_{p c}=\frac{l_{p c}^{+} \boldsymbol{n}_{p c}^{+}+l_{p c}^{-} \boldsymbol{n}_{p c}^{-}}{l_{p c}}, \quad l_{p c}=\left\|l_{p c}^{+} \boldsymbol{n}_{p c}^{+}+l_{p c}^{-} \boldsymbol{n}_{p c}^{-}\right\|_{2} .
$$

Finally, $\omega_{p c}$ denotes the subcell formed by $x_{c}, x_{p}, \frac{x_{p}+x_{p^{+}}}{2}$ and $\frac{x_{p}+x_{p^{-}}}{2}$. All the notations introduced are illustrated in Fig. 1. 


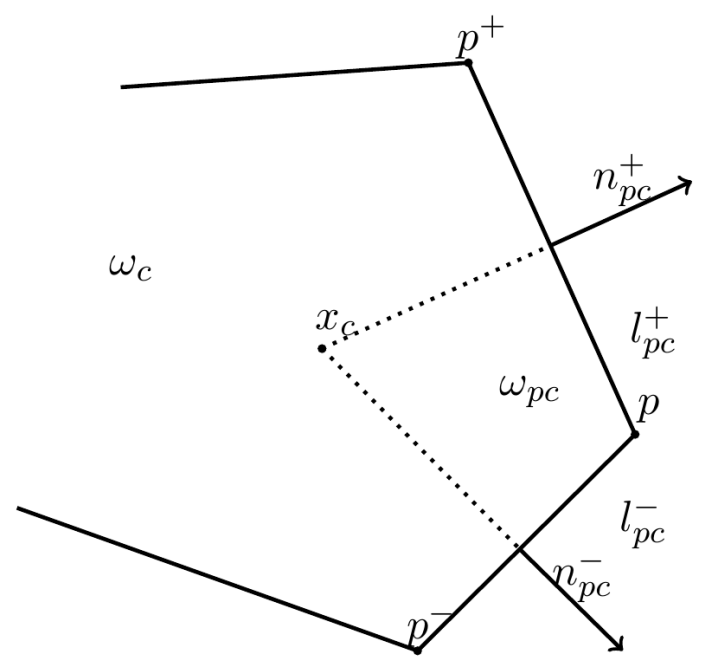

Figure 1: Cell notations.

\subsection{Nodal finite volume discretization}

Before deriving the numerical scheme, we first define the discrete variables for the quantities $\left\{\frac{1}{\rho}, u, \varepsilon_{i}, \varepsilon_{e}, \frac{E_{r}}{\rho}\right\}$ with a mean over the cell weighted by the density $\rho$. For any cell $\omega_{c}$ we set

$$
\phi_{c}=\frac{1}{m_{c}} \int_{\omega_{c}} \rho \phi \mathrm{d} x, \quad m_{c}=\int_{\omega_{c}} \rho \mathrm{d} x, \quad \forall \phi \in\left\{\frac{1}{\rho}, u, \varepsilon_{i}, \varepsilon_{e}, \frac{E_{r}}{\rho}\right\} .
$$

In this updated Lagrangian formalism the mesh moves with the matter so that the quantity $m_{c}$ associated to each cell $\omega_{c}$ does not depend on time. Consequently, integrating system (1.1) over a cell $\omega_{c}$ of mass $m_{c}$ and using the Reynold's formula directly leads to the following set of equations

$$
\left\{\begin{array}{l}
m_{c} \frac{\mathrm{d}}{\mathrm{d} t}\left(\frac{1}{\rho_{c}}\right)-\int_{\omega_{c}} \nabla \cdot \boldsymbol{u} \mathrm{d} \boldsymbol{x}=0, \\
m_{c} \frac{\mathrm{d} \boldsymbol{u}_{c}}{\mathrm{~d} t}+\int_{\omega_{c}} \nabla p \mathrm{~d} \boldsymbol{x}=0, \\
m_{c} \frac{\mathrm{d}}{\mathrm{d} t}\left(\frac{E_{r}}{\rho}\right)_{c}+\int_{\omega_{c}} p_{r} \nabla \cdot \boldsymbol{u} \mathrm{d} \boldsymbol{x}+\int_{\omega_{c}} \nabla \cdot \boldsymbol{F}_{r} \mathrm{~d} \boldsymbol{x}=c \int_{\omega_{c}} \sigma_{p}\left(a T_{e}^{4}-E_{r}\right) \mathrm{d} \boldsymbol{x}, \\
m_{c} \frac{\mathrm{d} \varepsilon_{e, c}}{\mathrm{~d} t}+\int_{\omega_{c}} p_{e} \nabla \cdot \boldsymbol{u} \mathrm{d} \boldsymbol{x}=c \int_{\omega_{c}} \sigma_{p}\left(E_{r}-a T_{e}^{4}\right) \mathrm{d} \boldsymbol{x}+\int_{\omega_{c}} \kappa\left(T_{i}-T_{e}\right) \mathrm{d} \boldsymbol{x}, \\
m_{c} \frac{\mathrm{d} \varepsilon_{i, c}}{d t}+\int_{\omega_{c}} p_{i} \nabla \cdot \boldsymbol{u} \mathrm{d} \boldsymbol{x}=\int_{\omega_{c}} \kappa\left(T_{e}-T_{i}\right) \mathrm{d} \boldsymbol{x} .
\end{array}\right.
$$


For the mass conservation equation, we apply the Green formula

$$
m_{c} \frac{\mathrm{d}}{\mathrm{d} t}\left(\frac{1}{\rho_{c}}\right)=\oint_{\partial \omega_{c}} \boldsymbol{u} \cdot \boldsymbol{n} \mathrm{d} l
$$

Now, assuming that the Lagrangian cell $\omega_{c}$ remains polygonal, $v_{c}$ only depends on the nodes positions defining the cell. Then, it is possible to show [19] that $\frac{\partial v_{c}}{\partial x_{p}}=l_{p c} \boldsymbol{n}_{p c}$. Consequently the equation rewrites

$$
\begin{aligned}
m_{c} \frac{\mathrm{d}}{\mathrm{d} t}\left(\frac{1}{\rho_{c}}\right)=\oint_{\partial \omega_{c}} \boldsymbol{u} \cdot \boldsymbol{n} \mathrm{d} l & =\sum_{p \in \mathcal{P}(c)} \frac{\partial v_{c}}{\partial x_{p}} \cdot \frac{\mathrm{d} \boldsymbol{x}_{p}}{\mathrm{~d} t} \\
& =\sum_{p \in \mathcal{P}(c)} l_{p c} \boldsymbol{n}_{p c} \cdot \boldsymbol{u}_{p} .
\end{aligned}
$$

Before proceeding to the analysis of the momentum conservation equation and the energy conservation equations, we first define sub-cell forces as follows

$$
f_{p c}^{\alpha}=-\int_{\partial \omega_{p c} \cap \partial \omega_{c}} p_{\alpha} n \mathrm{~d} l, \quad f_{p c}=-\int_{\partial \omega_{p c} \cap \partial \omega_{c}} p n \mathrm{~d} l=\sum_{\alpha} f_{p c^{\prime}}^{\alpha} \quad \forall \alpha \in\{e, i, r\} .
$$

For the momentum conservation, Green formula simply leads to

$$
m_{c} \frac{\mathrm{d} \boldsymbol{u}_{c}}{\mathrm{~d} t}=-\int_{\omega_{c}} \nabla p \mathrm{~d} \boldsymbol{x}=-\int_{\partial \omega_{c}} p \boldsymbol{n} \mathrm{d} l=\sum_{p \in \mathcal{P}(c)}-\int_{\partial \omega_{p c} \cap \partial \omega_{c}} p \boldsymbol{n d} l=\sum_{p \in \mathcal{P}(c)} f_{p c} .
$$

For the internal energy evolution equations, Green formula cannot be used directly since the equations are not written in a conservative form (internal energy formulation). To recover sub-cell forces expressions, one proceeds as follows for the ionic, electronic and radiation pressures

$$
-\int_{\omega_{c}} p_{\alpha} \nabla \cdot \boldsymbol{u} \mathrm{d} \boldsymbol{x}=-\int_{\omega_{c}} \nabla \cdot\left(p_{\alpha} \boldsymbol{u}\right) \mathrm{d} \boldsymbol{x}+\int_{\omega_{c}} \nabla p_{\alpha} \cdot \boldsymbol{u} \mathrm{d} x
$$

Assuming $\int_{\omega_{c}} \nabla p_{\alpha} \cdot \boldsymbol{u} \mathrm{d} \boldsymbol{x} \approx \boldsymbol{u}_{c} \cdot \int_{\omega_{c}} \nabla p_{\alpha} \mathrm{d} \boldsymbol{x}$ and using Green formula, Eq. (2.7) becomes

$$
\begin{aligned}
-\int_{\omega_{c}} p_{\alpha} \nabla \cdot \boldsymbol{u} \mathrm{d} x & =-\int_{\partial \omega_{c}} p_{\alpha} \boldsymbol{u} \cdot \boldsymbol{n} \mathrm{d} l+\boldsymbol{u}_{c} \cdot \int_{\omega_{c}} \nabla p_{\alpha} \mathrm{d} x \\
& =-\sum_{p \in \mathcal{P}(c)} \int_{\partial \omega_{p c} \cap \partial \omega_{c}} p_{\alpha} \boldsymbol{u} \cdot \boldsymbol{n} \mathrm{d} l+\boldsymbol{u}_{c} \cdot\left(\sum_{p \in \mathcal{P}(c)} \int_{\partial \omega_{p c} \cap \partial \omega_{c}} p_{\alpha} n \mathrm{~d} l\right) .
\end{aligned}
$$

At this point, considering the following approximation

$$
\int_{\partial \omega_{p c} \cap \partial \omega_{c}} p_{\alpha} \boldsymbol{u} \cdot \boldsymbol{n} \mathrm{d} l \approx \boldsymbol{u}_{p} \cdot \int_{\partial \omega_{p c} \cap \partial \omega_{c}} p_{\alpha} n \mathrm{~d} l
$$


Eq. (2.8) then reads

$$
\begin{aligned}
-\int_{\omega_{c}} p_{\alpha} \nabla \cdot \boldsymbol{u} \mathrm{d} \boldsymbol{x} & =-\sum_{p \in \mathcal{P}(c)} \boldsymbol{u}_{p} \cdot \int_{\partial \omega_{p c} \cap \partial \omega_{c}} p_{\alpha} n \mathrm{~d} l+\boldsymbol{u}_{c} \cdot\left(\sum_{p \in \mathcal{P}(c)} \int_{\partial \omega_{p c} \cap \partial \omega_{c}} p_{\alpha} n \mathrm{~d} l\right) \\
& =\sum_{p \in \mathcal{P}(c)} f_{p c}^{\alpha} \cdot\left(\boldsymbol{u}_{p}-\boldsymbol{u}_{c}\right) .
\end{aligned}
$$

The discretization of the radiation diffusion term is performed using a standard finite volume method. Indeed, it will been seen in the next section that, when radiation effects are considered, the numerical solution is projected onto the initial (Cartesian) mesh at each time step. We then consider

$$
\int_{\omega_{c}} \nabla \cdot \boldsymbol{F}_{r} \mathrm{~d} \boldsymbol{x}=\oint_{\partial \omega_{c}} \boldsymbol{F}_{r} \cdot \boldsymbol{n} \mathrm{d} l \approx \sum_{d \in \mathcal{N}(c)} \frac{c L_{d c}}{3 \sigma_{R, d c}} \frac{E_{r, c}-E_{r, d}}{\left\|x_{c}-x_{d}\right\|_{2}},
$$

where $\sigma_{R, d c}$ is the Rosseland opacity evaluated at the interface between the cells $\omega_{c}$ and $\omega_{d}$ and $L_{d c}$ is the length of the interface between the cells. The last point consists in discretizing the right hand side terms in system (2.2). If one considers a constant approximation on each cell the following semi-discrete scheme is obtained

$$
\left\{\begin{array}{l}
m_{c} \frac{\mathrm{d}}{\mathrm{d} t}\left(\frac{1}{\rho_{c}}\right)=\sum_{p \in \mathcal{P}(c)} l_{p c} \boldsymbol{n}_{p c} \cdot \boldsymbol{u}_{p}, \\
m_{c} \frac{\mathrm{d} \boldsymbol{u}_{c}}{\mathrm{~d} t}=\sum_{p \in \mathcal{P}(c)} f_{p c^{\prime}} \\
m_{c} \frac{\mathrm{d} \varepsilon_{e, c}}{\mathrm{~d} t}=\sum_{p \in \mathcal{P}(c)} f_{p c}^{e} \cdot\left(\boldsymbol{u}_{p}-\boldsymbol{u}_{c}\right)+c \sigma_{P, c} v_{c}\left(E_{r, c}-a T_{e, c}^{4}\right)+v_{c} \mathcal{K}_{c}\left(T_{i, c}-T_{e, c}\right), \\
m_{c} \frac{\mathrm{d} \varepsilon_{i, c}}{\mathrm{~d} t}=\sum_{p \in \mathcal{P}(c)} f_{p c}^{i} \cdot\left(\boldsymbol{u}_{p}-\boldsymbol{u}_{c}\right)+v_{c} \mathcal{K}_{c}\left(T_{e, c}-T_{i, c}\right), \\
m_{c} \frac{\mathrm{d}}{\mathrm{d} t}\left(\frac{E_{r}}{\rho}\right)_{c}=\sum_{p \in \mathcal{P}(c)} f_{p c}^{r} \cdot\left(\boldsymbol{u}_{p}-\boldsymbol{u}_{c}\right) \\
\quad+\sum_{d \in \mathcal{N}(c)} \frac{c L_{d c}}{3 \sigma_{R, d c}} \frac{E_{r, d}-E_{r, c}}{\left\|x_{c}-x_{d}\right\|_{2}}+c \sigma_{P, c} v_{c}\left(a T_{e, c}^{4}-E_{r, c}\right) .
\end{array}\right.
$$

In the next section the procedure leading to the definitions of the node velocities $u_{p}$ and $f_{p c}^{\alpha}$ is presented.

\subsection{Closure relations}

At this stage, the semi-discrete scheme (2.12) has to be completed with a procedure allowing to compute the sub-cell forces $f_{p c}$ and the node velocities $\boldsymbol{u}_{p}$. In the spirit of [20] this 
procedure is chosen to guarantee a correct total production entropy as well as to ensure the momentum and total energy conservation.

\section{Semi-discrete entropy production}

For ions and electrons, the semi-discrete entropy equation of the scheme (2.12) gives

$$
m_{c} T_{\alpha, c} \frac{d \eta_{c}^{\alpha}}{d t}=m_{c} \frac{d \varepsilon_{\alpha, c}}{d t}+m_{c} p_{c}^{\alpha} \frac{d}{d t}\left(\frac{1}{\rho_{c}}\right), \quad \forall \alpha \in\{e, i\} .
$$

Similarly for the photons the semi-discrete entropy equation reads

$$
m_{c} T_{r, c} \frac{d \eta_{c}^{r}}{d t}=m_{c} \frac{d}{d t}\left(\frac{E_{r}}{\rho}\right)_{c}+m_{c} p_{c}^{r} \frac{d}{d t}\left(\frac{1}{\rho_{c}}\right) .
$$

Now, from the semi-discrete relations (2.12) we have

$$
\left\{\begin{aligned}
m_{c} T_{i, c} \frac{\mathrm{d} \eta_{c}^{i}}{\mathrm{~d} t}= & \sum_{p \in \mathcal{P}(c)} \boldsymbol{f}_{p c}^{i} \cdot\left(\boldsymbol{u}_{p}-\boldsymbol{u}_{c}\right)+l_{p c} p_{c}^{i} \boldsymbol{n}_{p c} \cdot \boldsymbol{u}_{p}+v_{c} \mathcal{K}_{c}\left(T_{e, c}-T_{i, c}\right), \\
m_{c} T_{e, c} \frac{\mathrm{d} \eta_{c}^{e}}{\mathrm{~d} t}= & \sum_{p \in \mathcal{P}(c)} \boldsymbol{f}_{p c}^{e} \cdot\left(\boldsymbol{u}_{p}-\boldsymbol{u}_{c}\right)+l_{p c} p_{c}^{e} \boldsymbol{n}_{p c} \cdot \boldsymbol{u}_{p}+c \sigma_{P, c} v_{c}\left(E_{r, c}-a T_{e, c}^{4}\right) \\
& +v_{c} \mathcal{K}_{c}\left(T_{i, c}-T_{e, c}\right), \\
m_{c} T_{r, c} \frac{\mathrm{d} \eta_{c}^{r}}{\mathrm{~d} t}= & \sum_{p \in \mathcal{P}(c)} \boldsymbol{f}_{p c}^{r} \cdot\left(\boldsymbol{u}_{p}-\boldsymbol{u}_{c}\right)+l_{p c} p_{c}^{r} \boldsymbol{n}_{p c} \cdot \boldsymbol{u}_{p}+\sum_{d \in \mathcal{N}(c)} \frac{c L_{d c}}{3 \sigma_{R, d c}} \frac{E_{r, d}-E_{r, c}}{\left\|x_{c}-x_{d}\right\|_{2}} \\
& +c \sigma_{P, c} v_{c}\left(a T_{e, c}^{4}-E_{r, c}\right) .
\end{aligned}\right.
$$

Since the integral of the normal over a closed contour is zero, we then have for all cells $\omega_{c}$

$$
\int_{\partial \omega_{c}} n \mathrm{~d} l=\sum_{p \in \mathcal{P}(c)} l_{p c} \boldsymbol{n}_{p c}=\mathbf{0}, \quad \sum_{p \in \mathcal{P}(c)} l_{p c} p_{c}^{\alpha} \boldsymbol{u}_{c} \cdot \boldsymbol{n}_{p c}=0, \quad \forall \alpha \in\{r, e, i\},
$$

so that the set of equations (2.15) becomes

$$
\left\{\begin{aligned}
m_{c} T_{r, c} \frac{\mathrm{d} \eta_{c}^{r}}{\mathrm{~d} t}= & \sum_{p \in \mathcal{P}(c)}\left(f_{p c}^{r}+l_{p c} p_{c}^{r} \boldsymbol{n}_{p c}\right) \cdot\left(\boldsymbol{u}_{p}-\boldsymbol{u}_{c}\right)+\sum_{d \in \mathcal{N}(c)} \frac{c L_{d c}}{3 \sigma_{R, d c}} \frac{E_{r, d}-E_{r, c}}{\left\|x_{c}-x_{d}\right\|_{2}} \\
& +c \sigma_{P, c} v_{c}\left(a T_{e, c}^{4}-E_{r, c}\right), \\
m_{c} T_{e, c} \frac{\mathrm{d} \eta_{c}^{e}}{\mathrm{~d} t}= & \sum_{p \in \mathcal{P}(c)}\left(f_{p c}^{e}+l_{p c} p_{c}^{e} \boldsymbol{n}_{p c}\right) \cdot\left(\boldsymbol{u}_{p}-\boldsymbol{u}_{c}\right)+c \sigma_{P, c} v_{c}\left(E_{r, c}-a T_{e, c}^{4}\right) \\
& \quad+v_{c} \mathcal{K}_{c}\left(T_{i, c}-T_{e, c}\right), \\
m_{c} T_{i, c} \frac{\mathrm{d} \eta_{c}^{i}}{\mathrm{~d} t}= & \sum_{p \in \mathcal{P}(c)}\left(f_{p c}^{i}+l_{p c} p_{c}^{i} \boldsymbol{n}_{p c}\right) \cdot\left(\boldsymbol{u}_{p}-\boldsymbol{u}_{c}\right)+v_{c} \mathcal{K}_{c}\left(T_{e, c}-T_{i, c}\right) .
\end{aligned}\right.
$$


At this point, a sufficient condition to ensure the second principle of thermodynamics for the semi-discrete model is to define the sub-cell forces as follows

$$
\boldsymbol{f}_{p c}^{\alpha}=-l_{p c} p_{c}^{\alpha} \boldsymbol{n}_{p c}+\boldsymbol{M}_{p c}^{\alpha}\left(\boldsymbol{u}_{p}-\boldsymbol{u}_{c}\right), \quad \forall \alpha \in\{r, e, i\},
$$

where $\boldsymbol{M}_{p c}^{\alpha}$ is a positive semi-positive matrix named in the following as a dissipation matrix. Now, in order to enforce a correct discrete total entropy production, we extend the standard dissipation matrix definition [20] and choose the following definitions

$$
\left\{\begin{array}{l}
\boldsymbol{M}_{p c}^{e}=\rho_{e} c_{s}\left(l_{p c}^{+} \boldsymbol{n}_{p c}^{+} \otimes \boldsymbol{n}_{p c}^{+}+l_{p c}^{-} \boldsymbol{n}_{p c}^{-} \otimes \boldsymbol{n}_{p c}^{-}\right), \\
\boldsymbol{M}_{p c}^{i}=\rho_{i} c_{s}\left(l_{p c}^{+} \boldsymbol{n}_{p c}^{+} \otimes \boldsymbol{n}_{p c}^{+}+l_{p c}^{-} \boldsymbol{n}_{p c}^{-} \otimes \boldsymbol{n}_{p c}^{-}\right),
\end{array}\right.
$$

where $\rho_{e}, \rho_{i}, c_{S}$ are respectively the density of electrons, the density of ions and the speed of sound. In order to compute $\rho_{e}$ and $\rho_{i}$ one considers the definition of the total density and the quasi-neutrality relation

$$
\rho=\rho_{e}+\rho_{i}=m_{e} n_{e}+m_{i} n_{i}, \quad n_{e}=Z n_{i}
$$

where $n_{e}$ and $n_{i}$ are respectively the electronic and ionic density, $m_{e}$ and $m_{i}$ the electron and ion mass and $Z$ the atomic number of the species. These relations rewrite

$$
\rho_{e}=\frac{Z \frac{m_{e}}{m_{i}}}{1+Z \frac{m_{e}}{m_{i}}} \rho, \quad \rho_{i}=\frac{1}{1+Z \frac{m_{e}}{m_{i}}} \rho,
$$

so that in the limit $m_{e} / m_{i}$ tends to zero, one recovers the infinite mass ratio conditions $\rho_{e}=0$ and $\rho_{i}=\rho$. One understands here that the choice (2.19) for the dissipation matrices enables to enforce at the discrete level an quasi-isentropic electron transport while the total entropy production is mainly due to ions as described in [34]. In addition, we consider that there is no entropy production due to photons in the shock wave. We then define the radiation dissipation matrix

$$
\boldsymbol{M}_{p c}^{r}=\mathbf{0}
$$

Discussion. We point out that in the limit $m_{e} / m_{i}$ tends to zero there is no numerical viscosity on the electron and photon energy equation. This is different from standard Godunov-type approaches in which the numerical viscosity is put on all equations leading to strong stability properties but dissipating on both electron and ion (even in the limit $m_{e} / m_{i}$ tends to zero). With the present choice one could expect stability issues (when all the internal energy is put on electrons for example). However, it will be shown in the numerical section, that the resulting scheme remains perfectly stable in all the numerical tests performed. 


\section{Momentum and total energy conservation}

From the semi-discrete scheme equations (2.12), a direct summation leads to

$$
\begin{aligned}
& m_{c} \frac{\mathrm{d}}{\mathrm{d} t}\left(\left(\frac{E_{r}}{\rho}\right)_{c}+\varepsilon_{e, c}+\varepsilon_{i, c}+\frac{1}{2}\left\|\boldsymbol{u}_{c}\right\|_{2}^{2}\right) \\
= & \sum_{p \in \mathcal{P}(c)} f_{p c} \cdot\left(\boldsymbol{u}_{p}-\boldsymbol{u}_{c}\right)+\sum_{d \in \mathcal{N}(c)} \frac{c L_{d c}}{3 \sigma_{R, d c}} \frac{E_{r, d}-E_{r, c}}{\left\|x_{c}-x_{d}\right\|_{2}} \\
= & \sum_{p \in \mathcal{P}(c)} \boldsymbol{f}_{p c} \cdot \boldsymbol{u}_{p}+\sum_{d \in \mathcal{N}(c)} \frac{c L_{d c}}{3 \sigma_{R, d c}} \frac{E_{r, d}-E_{r, c}}{\left\|x_{c}-x_{d}\right\|_{2}} .
\end{aligned}
$$

Now, neglecting possible boundary conditions contribution and adding the conservation of momentum and total energy equations over the whole space domain gives

$$
\left\{\begin{array}{l}
\sum_{c} m_{c} \frac{\mathrm{d} \boldsymbol{u}_{c}}{\mathrm{~d} t}=\sum_{c} \sum_{p \in \mathcal{P}(c)} f_{p c^{\prime}} \\
\sum_{c} m_{c} \frac{\mathrm{d}}{\mathrm{d} t}\left(\left(\frac{E_{r}}{\rho}\right)_{c}+\varepsilon_{e, c}+\varepsilon_{i, c}+\frac{1}{2}\left\|\boldsymbol{u}_{c}\right\|^{2}\right) \\
\quad=\sum_{c}\left(\sum_{p \in \mathcal{P}(c)} \boldsymbol{f}_{p c} \cdot \boldsymbol{u}_{p}+\sum_{d \in \mathcal{N}(c)} \frac{c L_{d c}}{3 \sigma_{R, d c}} \frac{E_{r, d}-E_{r, c}}{\left\|x_{c}-x_{d}\right\|_{2}}\right) .
\end{array}\right.
$$

After inverting the node and cell sums, a sufficient condition to ensure the momentum and total energy discrete conservation is

$$
\sum_{c \in \mathcal{C}(p)} f_{p c}=\mathbf{0}
$$

Finally the expression of the sub-cell forces leads to the following definition for the node velocity

$$
\boldsymbol{u}_{p}=\left(\sum_{c \in \mathcal{C}(p)}\left(\boldsymbol{M}_{p c}^{r}+\boldsymbol{M}_{p c}^{e}+\boldsymbol{M}_{p c}^{i}\right)\right)^{-1} \sum_{c \in \mathcal{C}(p)} l_{p c} p_{c} \boldsymbol{n}_{p c}+\left(\boldsymbol{M}_{p c}^{r}+\boldsymbol{M}_{p c}^{e}+\boldsymbol{M}_{p c}^{i}\right) \boldsymbol{u}_{c}
$$

\subsection{Time discretisation and CFL condition}

Standard Euler forward strategies are finally considered for the hydrodynamics part while, because of the stiffness associated to the radiation terms, the radiation diffusion 
term and the coupling terms are chosen implicit

$$
\left\{\begin{aligned}
\frac{m_{c}}{\Delta t}\left(\frac{E_{r, c}^{n+1}}{\rho_{c}^{n+1}}-\frac{E_{r, c}^{n}}{\rho_{c}^{n}}\right)= & \sum_{p \in \mathcal{P}(c)} \boldsymbol{f}_{p c}^{r, n} \cdot\left(\boldsymbol{u}_{p}^{n}-\frac{\boldsymbol{u}_{c}^{n+1}+\boldsymbol{u}_{c}^{n}}{2}\right) \\
& +\sum_{d \in \mathcal{N}(c)} \frac{c L_{d c}}{3 \sigma_{R, d c}^{n+1}} \frac{E_{r, d}^{n+1}-E_{r, c}^{n+1}}{\left\|x_{c}-x_{d}\right\|_{2}}+c \sigma_{P, c}^{n+1} v_{c}\left(a\left(T_{e, c}^{n+1}\right)^{4}-E_{r, c}^{n+1}\right), \\
\frac{m_{c}}{\Delta t}\left(\varepsilon_{e, c}^{n+1}-\varepsilon_{e, c}^{n}\right)= & \sum_{p \in \mathcal{P}(c)} f_{p c}^{e, n} \cdot\left(\boldsymbol{u}_{p}^{n}-\frac{\boldsymbol{u}_{c}^{n+1}+\boldsymbol{u}_{c}^{n}}{2}\right)+c \sigma_{P, c}^{n+1} v_{c}\left(E_{r, c}^{n+1}-a\left(T_{e, c}^{n+1}\right)^{4}\right) \\
& +v_{c} \kappa_{c}^{n+1}\left(T_{i, c}^{n+1}-T_{e, c}^{n+1}\right), \\
\frac{m_{c}}{\Delta t}\left(\varepsilon_{i, c}^{n+1}-\varepsilon_{i, c}^{n}\right)= & \sum_{p \in \mathcal{P}(c)} f_{p c}^{i, n} \cdot\left(\boldsymbol{u}_{p}^{n}-\frac{\boldsymbol{u}_{c}^{n+1}+\boldsymbol{u}_{c}^{n}}{2}\right)+v_{c} \kappa_{c}^{n+1}\left(T_{e, c}^{n+1}-T_{i, c}^{n+1}\right) .
\end{aligned}\right.
$$

Remark 2.1. Notice here that the temporal discretization of the velocity divergence is asymmetric, where $u_{p}$ is taken from the time level $n$ and $u_{c}$ from the average of $n+1$ and $n$. This choice is made in order to enforce an exact conservation of the discrete total energy (see the proof in next section). However, this implies that a divergence-free velocity function can produce mechanical work. Though the quantitative effects might be negligible in most cases this maybe a disadvantage of the scheme when studying incompressible flows. Of course $u_{c}$ may also be taken at the time level $n$ to avoid this drawback. Here the full discrete energy conservation is preferred.

In order to deal with the explicit part (hydrodynamics part) the following CFL condition is considered

$$
\frac{\Delta t c_{s, c}}{v_{c}}\left(\sum_{p \in \mathcal{P}(c)} \frac{l_{p c}^{+}+l_{p c}^{-}}{2}\right) \leq \frac{1}{2} .
$$

The set of equations (2.27) is nonlinear and may be solved with a standard NewtonRaphson type procedure. However, such a strategy does not ensure a control of the temperatures (positiveness of the discrete temperatures) during all the iterative process. Consequently, in the next section, extending the ideas proposed in [8], a convex combination based scheme is introduced.

\subsection{Numerical approach for radiation diffusion and coupling terms}

System (2.27) may be reformulated in order to derive convex combination based schemes ensuring strong stability properties. Introducing the quantities $\phi_{\alpha}$

$$
\phi_{\alpha}=a T_{\alpha}^{4}, \quad \forall \alpha \in\{r, e, i\},
$$


and the coefficients $\beta_{\alpha}$ and $\delta_{i e}$ defined by

$$
\beta_{\alpha}^{n+1}=\frac{\phi_{\alpha}^{n+1}-\phi_{\alpha}^{n}}{\varepsilon_{\alpha}^{n+1}-\varepsilon_{\alpha}^{n}}, \quad \delta_{i e}^{n+1}=\frac{T_{i}^{n+1}-T_{e}^{n+1}}{\phi_{e}^{n+1}-\phi_{i}^{n+1}}, \quad \forall \alpha \in\{e, i\},
$$

system (2.27) may be rewrites as follows

$$
\left\{\begin{aligned}
\frac{m_{c}}{\Delta t}\left(\frac{\phi_{r, c}^{n+1}}{\rho_{c}^{n+1}}-\frac{\phi_{r, c}^{n}}{\rho_{c}^{n}}\right)= & \sum_{p \in \mathcal{P}(c)} f_{p c}^{r, n} \cdot\left(\boldsymbol{u}_{p}^{n}-\frac{\boldsymbol{u}_{c}^{n+1}+\boldsymbol{u}_{c}^{n}}{2}\right)+\sum_{d \in \mathcal{N}(c)} \frac{c L_{d c}}{3 \sigma_{R, d c}^{n+1}} \frac{\phi_{r, d}^{n+1}-\phi_{r, c}^{n+1}}{\left\|x_{c}-x_{d}\right\|_{2}} \\
& +c \sigma_{P, c}^{n+1} v_{c}\left(\phi_{e, c}^{n+1}-\phi_{r, c}^{n+1}\right), \\
\frac{m_{c}}{\Delta t \beta_{e, c}^{n+1}}\left(\phi_{e, c}^{n+1}-\phi_{e, c}^{n}\right)= & \sum_{p \in \mathcal{P}(c)} f_{p c}^{e, n} \cdot\left(\boldsymbol{u}_{p}^{n}-\frac{\boldsymbol{u}_{c}^{n+1}+\boldsymbol{u}_{c}^{n}}{2}\right)+c \sigma_{P, c}^{n+1} v_{c}\left(\phi_{r, c}^{n+1}-\phi_{e, c}^{n+1}\right) \\
& +v_{c} \kappa_{c}^{n+1} \delta_{i e, c}^{n+1}\left(\phi_{i, c}^{n+1}-\phi_{e, c}^{n+1}\right), \\
\frac{m_{c}}{\Delta t \beta_{i, c}^{n+1}}\left(\phi_{i, c}^{n+1}-\phi_{i, c}^{n}\right)= & \sum_{p \in \mathcal{P}(c)} f_{p c}^{i, n} \cdot\left(\boldsymbol{u}_{p}^{n}-\frac{\boldsymbol{u}_{c}^{n+1}+\boldsymbol{u}_{c}^{n}}{2}\right)+v_{c} \mathcal{K}_{c}^{n+1} \delta_{i e, c}^{n+1}\left(\phi_{e, c}^{n+1}-\phi_{i, c}^{n+1}\right) .
\end{aligned}\right.
$$

Note at this point, system (2.30) is still non-linear because of the implicit time discretization of the opacities, the electron-ion coupling term, $\beta_{\alpha}$ and $\delta_{i e}$. The scheme is now rewritten under convex combination form, which is solved with a standard fix point procedure. This procedure is initialized setting

$$
\phi_{\alpha}^{n+1,0}=\phi_{\alpha}^{n}, \quad \forall \alpha \in\{r, e, i\},
$$

and denoting the subiteration with an index $k$. The resulting iterative scheme writes

$$
\begin{aligned}
& \frac{m_{c}}{\Delta t}\left(\frac{\phi_{r, c}^{n+1, k+1}}{\rho_{c}^{n+1}}-\frac{\phi_{r, c}^{n}}{\rho_{c}^{n}}\right)=\sum_{p \in \mathcal{P}(c)} f_{p c}^{r, n} \cdot\left(\boldsymbol{u}_{p}^{n}-\frac{\boldsymbol{u}_{c}^{n+1}+\boldsymbol{u}_{c}^{n}}{2}\right) \\
& +\sum_{d \in \mathcal{N}(c)} \frac{c L_{d c}}{3 \sigma_{R, d c}^{n+1, k}} \frac{\phi_{r, d}^{n+1, k+1}-\phi_{r, c}^{n+1, k+1}}{\left\|x_{c}-x_{d}\right\|_{2}}+c \sigma_{P, c}^{n+1, k} v_{c}\left(\phi_{e, c}^{n+1, k+1}-\phi_{r, c}^{n+1, k+1}\right), \\
& \left\{\frac{m_{c}}{\Delta t \beta_{e, c}^{n+1, k}}\left(\phi_{e, c}^{n+1, k+1}-\phi_{e, c}^{n}\right)=\sum_{p \in \mathcal{P}(c)} f_{p c}^{e, n} \cdot\left(\boldsymbol{u}_{p}^{n}-\frac{\boldsymbol{u}_{c}^{n+1}+\boldsymbol{u}_{c}^{n}}{2}\right)\right. \\
& +c \sigma_{P, c}^{n+1, k} v_{c}\left(\phi_{r, c}^{n+1, k+1}-\phi_{e, c}^{n+1, k+1}\right)+v_{c} \kappa_{c}^{n+1, k} \delta_{i e, c}^{n+1, k}\left(\phi_{i, c}^{n+1, k+1}-\phi_{e, c}^{n+1, k+1}\right), \\
& \frac{m_{c}}{\Delta t \beta_{i, c}^{n+1, k}}\left(\phi_{i, c}^{n+1, k+1}-\phi_{i, c}^{n}\right)=\sum_{p \in \mathcal{P}(c)} f_{p c}^{i, n} \cdot\left(\boldsymbol{u}_{p}^{n}-\frac{\boldsymbol{u}_{c}^{n+1}+\boldsymbol{u}_{c}^{n}}{2}\right) \\
& +v_{c} \kappa_{c}^{n+1, k} \delta_{i e, c}^{n+1, k}\left(\phi_{e, c}^{n+1, k+1}-\phi_{i, c}^{n+1, k+1}\right) \text {, }
\end{aligned}
$$


where

$$
\beta_{\alpha}^{n+1, k}=\frac{\phi_{\alpha}^{n+1, k}-\phi_{\alpha}^{n}}{\varepsilon_{\alpha}^{n+1, k}-\varepsilon_{\alpha}^{n}}, \quad \delta_{i e}^{n+1, k}=\frac{T_{i}^{n+1, k}-T_{e}^{n+1, k}}{\phi_{e}^{n+1, k}-\phi_{i}^{n+1, k}}, \quad \forall \alpha \in\{e, i\} .
$$

so that the scheme finally writes under the following convex combination form

$$
\left\{\begin{aligned}
& \phi_{i, c}^{n+1, k+1}=h_{c}^{n+1, k} \psi_{i, c}^{n+1, k}+\left(1-h_{c}^{n+1, k}\right) \phi_{e, c}^{n+1, k+1}, \\
& \phi_{e, c}^{n+1, k+1}=f_{c}^{n+1, k}\left(g_{c}^{n+1, k} \psi_{e, c}^{n+1, k}+\left(1-g_{c}^{n+1, k}\right) \psi_{i, c}^{n+1, k}\right)+\left(1-f_{c}^{n+1, k}\right) \phi_{r, c}^{n+1, k+1}, \\
& \phi_{r, c}^{n+1, k+1}\left(\frac{m_{c}}{\rho_{c}^{n+1}}+\Delta t c \sigma_{P, c}^{n+1, k} f_{c}^{n+1, k} v_{c}\right)+\sum_{d \in \mathcal{N}(c)} \frac{c \Delta t L_{d c}}{3 \sigma_{R, d c}^{n+1, k}} \frac{\phi_{r, c}^{n+1, k+1}-\phi_{r, d}^{n+1, k+1}}{\left\|x_{c}-x_{d}\right\|_{2}} \\
&=\psi_{r, c}^{n+1, k} m_{c}+c v_{c} \sigma_{P, c}^{n+1, k} \Delta t f_{c}^{n+1, k}\left(g_{c}^{n+1, k} \psi_{e, c}^{n+1, k}+\left(1-g_{c}^{n+1, k}\right) \psi_{i, c}^{n+1, k}\right),
\end{aligned}\right.
$$

where the following notations have been used

$$
\begin{aligned}
h_{c}^{n+1, k} & =\frac{1}{1+v_{c} c \beta_{i, c}^{n+1, k} \kappa_{c}^{n+1, k} \delta_{i e, c}^{n+1, k} \Delta t / m_{c}}, \quad g_{c}^{n+1, k} \frac{1}{1+h_{c}^{n+1, k} \delta_{i e, c}^{n+1, k} v_{c} c \kappa_{c}^{n+1, k} \beta_{e, c}^{n+1, k} \Delta t / m_{c}}, \\
f_{c}^{n+1, k} & =\frac{1}{1+g_{c}^{n+1, k} v_{c} c \sigma_{P, c}^{n+1, k} \beta_{e, c}^{n+1, k} \Delta t / m_{c}},
\end{aligned}
$$

in addition to the hydrodynamics terms

$$
\psi_{\alpha, c}^{n+1, k}=\phi_{\alpha, c}^{n}+\frac{\beta_{\alpha, c}^{n+1, k} \Delta t}{m_{c}} \sum_{p \in \mathcal{P}(c)} f_{p c}^{\alpha, n} \cdot\left(\boldsymbol{u}_{p}^{n}-\frac{\boldsymbol{u}_{c}^{n+1}+\boldsymbol{u}_{c}^{n}}{2}\right), \quad \forall \alpha \in\{e, i\},
$$

and

$$
\psi_{r, c}^{n+1, k}=\frac{\phi_{r, c}^{n}}{\rho_{c}^{n}}+\frac{\Delta t}{m_{c}} \sum_{p \in \mathcal{P}(c)} f_{p c}^{r, n} \cdot\left(\boldsymbol{u}_{p}^{n}-\frac{\boldsymbol{u}_{c}^{n+1}+\boldsymbol{u}_{c}^{n}}{2}\right) .
$$

In the next section, the main scheme properties are detailed.

\subsection{Numerical properties}

In this section the positiveness of the temperatures, the discrete entropy production and discrete total energy conservation are proved.

Property 2.1 (Temperatures positiveness). The positiveness of $\psi_{r, c}^{n+1, k}, \psi_{e, c}^{n+1, k}$ and $\psi_{i, c}^{n+1, k}$ is ensured at each sub-iteration $k$. 
Proof. This property directly comes from the reformulation procedure and the fix point algorithm. Indeed the third equation of system (2.33) rewrites

$$
\left\{\begin{aligned}
\phi_{r, c}^{n+1, k+1}\left(\frac{m_{c}}{\rho_{c}^{n+1}}+\Delta t c \sigma_{P, c}^{n+1, k} f_{c}^{n+1, k} v_{c}\right. & \left.+\sum_{d \in \mathcal{N}(c)} \frac{1}{3 \sigma_{R, d c}^{n+1, k}} \frac{c \Delta t L_{d c}}{\left\|x_{c}-x_{d}\right\|_{2}}\right) \\
& -\sum_{d \in \mathcal{N}(c)} \frac{c \Delta t L_{d c}}{3 \sigma_{R, d c}^{n+1, k}} \frac{\phi_{r, d}^{n+1, k+1}}{\left\|x_{c}-x_{d}\right\|_{2}} \\
=\psi_{r, c}^{n+1, k} m_{c}+c v_{c} \sigma_{P, c}^{n+1, k} \Delta t f_{c}^{n+1, k} & \left(g_{c}^{n+1, k} \psi_{e, c}^{n+1, k}+\left(1-g_{c}^{n+1, k}\right) \psi_{i, c}^{n+1, k}\right) .
\end{aligned}\right.
$$

Solving this last system comes down to the resolution of a linear system whose associated matrix is a M-matrix and the components of the right-hand side are all positive thanks to the convex combination form. Consequently, the unknowns $\phi_{r, c}^{n+1, k+1}$ are all positive. Finally, remarking that the two first equations of system (2.33) also writes as convex combinations, one eventually gets the positiveness of $\phi_{e, c}^{n+1, k+1}$ and $\phi_{i, c}^{n+1, k+1}$.

Remark 2.2. The positiveness of $\psi_{r, c}^{n+1, k}, \psi_{e, c}^{n+1, k}$ and $\psi_{i, c}^{n+1, k}$ is ensured as soon as the time step $\Delta t$ is chosen such that

$$
\left\{\begin{array}{l}
\phi_{i, c}^{n}+\frac{\beta_{i, c}^{n+1, k} \Delta t}{m_{c}} \sum_{p \in \mathcal{P}(c)} f_{p c}^{i, n} \cdot\left(\boldsymbol{u}_{p}^{n}-\frac{\boldsymbol{u}_{c}^{n+1}+\boldsymbol{u}_{c}^{n}}{2}\right) \geq 0 \\
\phi_{e, c}^{n}+\frac{\beta_{e, c}^{n+1, k} \Delta t}{m_{c}} \sum_{p \in \mathcal{P}(c)} f_{p c}^{e, n} \cdot\left(\boldsymbol{u}_{p}^{n}-\frac{\boldsymbol{u}_{c}^{n+1}+\boldsymbol{u}_{c}^{n}}{2}\right) \geq 0 \\
\frac{\phi_{r, c}^{n}}{\rho_{c}^{n}}+\frac{\Delta t}{m_{c}} \sum_{p \in \mathcal{P}(c)} f_{p c}^{r, n} \cdot\left(\boldsymbol{u}_{p}^{n}-\frac{\boldsymbol{u}_{c}^{n+1}+\boldsymbol{u}_{c}^{n}}{2}\right) \geq 0
\end{array}\right.
$$

Thanks to the implicit-explicit strategy the constraints on the time step $\Delta t$ are only driven by the hydrodynamics part which is not stiff.

Property 2.2 (Semi-discrete entropy production). The total semi-discrete entropy in the domain is dissipated

$$
\sum_{c} m_{c} \frac{\mathrm{d} \eta_{c}}{\mathrm{~d} t} \geq 0
$$

Proof. The proof is given in Appendix.

Property 2.3 (Discrete total energy conservation). The total discrete energy is conserved

$$
\sum_{c} m_{c}\left(\frac{\phi_{r, c}^{n+1}}{\rho_{c}^{n+1}}+\varepsilon_{e, c}^{n+1}+\varepsilon_{i, c}^{n+1}+\frac{\left\|\boldsymbol{u}_{c}^{n+1}\right\|^{2}}{2}\right)=\sum_{c} m_{c}\left(\frac{\phi_{r, c}^{n}}{\rho_{c}^{n}}+\varepsilon_{e, c}^{n}+\varepsilon_{i, c}^{n}+\frac{\left\|\boldsymbol{u}_{c}^{n}\right\|^{2}}{2}\right) .
$$

Proof. The proof is given in Appendix. 


\subsubsection{Remap procedure and CFL condition}

It is well-known when working with the updated Lagrangian formalism, that the mesh is subject to strong deformations as it moves according to the fluid velocity. This motivates a regularisation phase (projection phase) in which the numerical solution is projected onto the initial (Cartesian) mesh. The resulting numerical strategy is referred in the literature as Lagrange-remap strategy [21]. In addition, working with a simple (Cartesian) mesh enables a straightforward discretization of the diffusion terms, applying the diffusion operator on the Eulerian cartesian mesh. The remap procedure (projection step) considered here is standard [21] and the Lagrangian CFL condition (2.28) is replaced by the following one

$$
\frac{\Delta t}{v_{c}}\left(\left(\left\|u_{c}^{n}\right\|+c_{s, c}\right) \sum_{p \in \mathcal{P}(c)} \frac{l_{p c}^{+}+l_{p c}^{-}}{2}\right) \leq \frac{1}{2}
$$

In the next section, pure hydrodynamics and radiation hydrodynamics numerical tests are presented. As a consequence of the above discussion, pure hydrodynamics tests are performed in Lagrangian formalism (no remap phase) to study the robustness of the scheme (recall here that there is no numerical viscosity on the electron and photon energy equations) while radiation-hydrodynamics tests are carried out with a second order remap projection step on the initial mesh. In addition, since the matrix associated to the resolution of linear system (2.37) is symmetric, a conjugate gradient algorithm coupled with a Jacobi preconditionner (diagonal preconditionner) is chosen. For a given threshold $\varepsilon$, the iterative procedure is carried out until the gap between two iterations becomes small enough

$$
\max _{\alpha \in\{e, i, r\}}\left\|\frac{\phi_{\alpha, c}^{n+1, k+1}-\phi_{\alpha, c}^{n+1, k}}{\phi_{\alpha, c}^{n+1, k+1}+\phi_{\alpha, c}^{n+1, k}}\right\|_{\infty} \leq \varepsilon .
$$

\section{Numerical results}

In this section, hydrodynamics, radiation and radiation-hydrodynamics test cases are presented. The numerical behavior of the scheme is studied in various physical regimes and compared with reference solutions.

\subsection{Hydrodynamic test cases}

\subsubsection{Two temperature hydrodynamics shock wave}

In order to study the electron and ion temperature decoupling as a shock wave propagates in the plasma medium, consider a simple 1D compression problem. At the left boundary of the domain the plasma is compressed by a moving piston resulting in the propagation of a right-going shock wave. This configuration is displayed in Fig. 2. The 


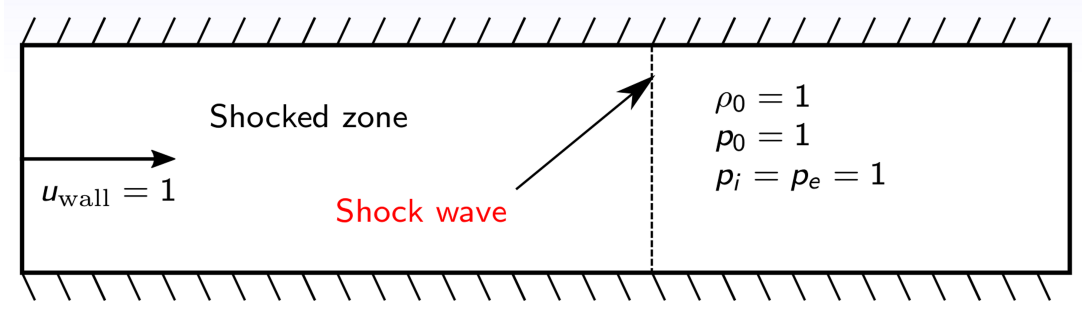

Figure 2: Piston configuration studied. All the details are described in the text.
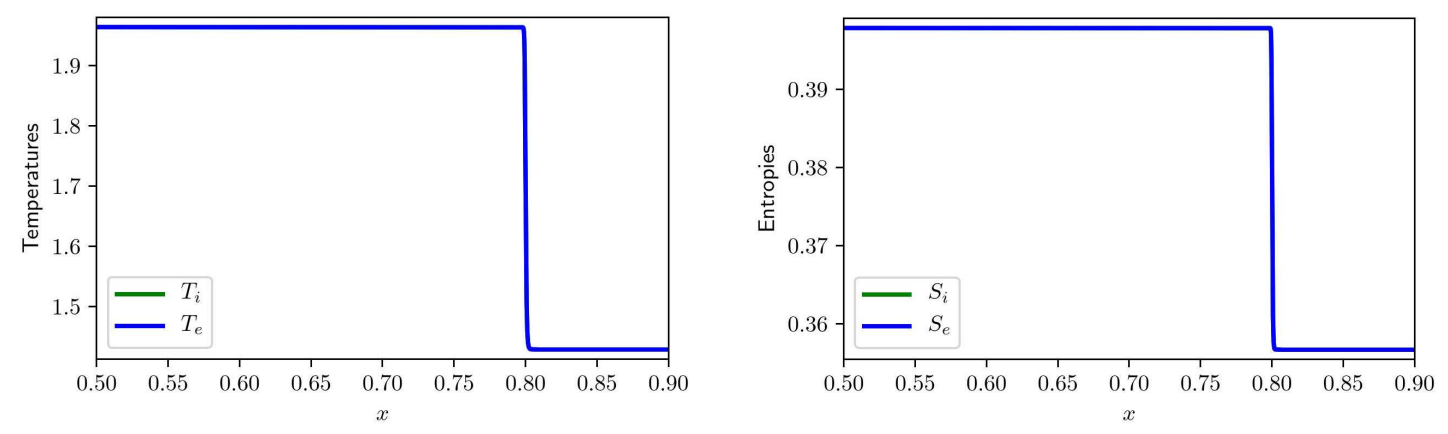

Figure 3: Temperature (on the left) and entropy (on the right) for the piston shock wave test case at time 0.4 in the case $m_{i} / m_{e}=1$.

space domain is set to $[0,1]$ and the initial conditions are the following

$$
\left(\begin{array}{l}
\rho \\
p \\
u
\end{array}\right)(t=0)=\left(\begin{array}{l}
1 \\
1 \\
0
\end{array}\right)
$$

and a constant velocity equal to 1 is enforced at the left boundary. Consider a diatomic perfect gas $(\gamma=1.4)$ in a one dimension space. The heat capacities for ions and electrons are taken equal to 1 and the relaxation coefficient is fixed to $\kappa=20$. The ratio between electron and ion masses is set to $1 / 2000$. We choose 2000 cells and a CFL number equal to 0.45 . In Figs. 3 to 5, the temperature and entropy fields (in the case of an ideal case closure by $S_{\alpha}=C_{v} \ln \left(T_{\alpha} \rho^{1-\gamma}\right)$ ) are displayed at time $t=0.4$ for different electron-ion mass ratios. In the case of a physically relevant ratio, (for example taking $m_{i} / m_{e}=2000$ ), it is recovered that the entropy production is mainly due to the ion contribution. After the shock, due to the fact that the entropy deposition is greater on the ions than on the electrons, we observe a coupling of the temperatures. Further from the shock, the temperatures are balancing due to the coupling term. Since the ions are heavier than the electrons, they dissipate more entropy. Finally, we observe a wall heating phenomenon on the left boundary which is a classical drawback of Lagrangian scheme. 

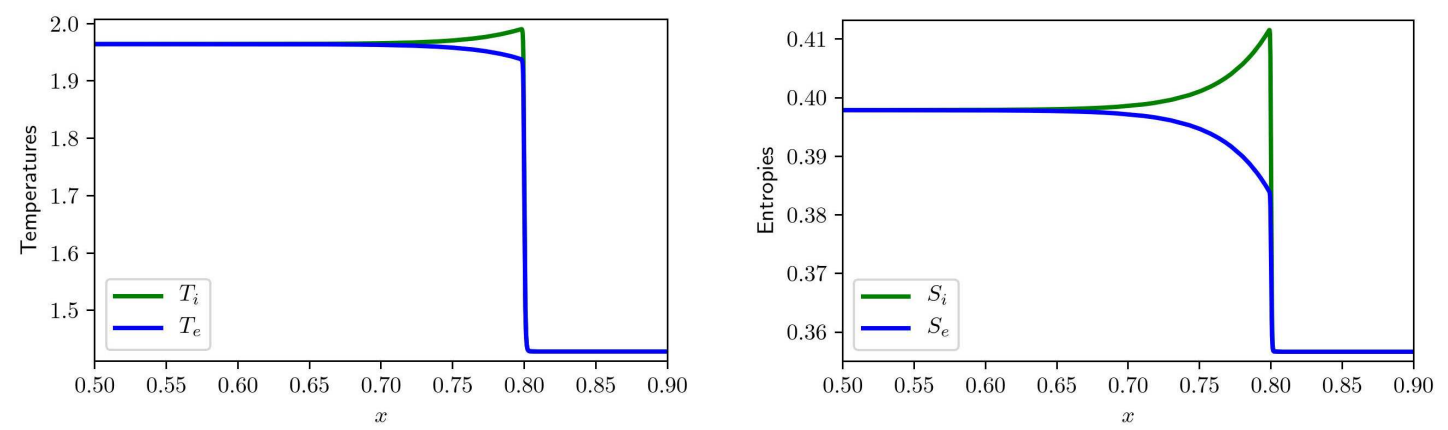

Figure 4: Temperature (on the left) and entropy (on the right) for the piston shock wave test case at time 0.4 in the case $m_{i} / m_{e}=2$.
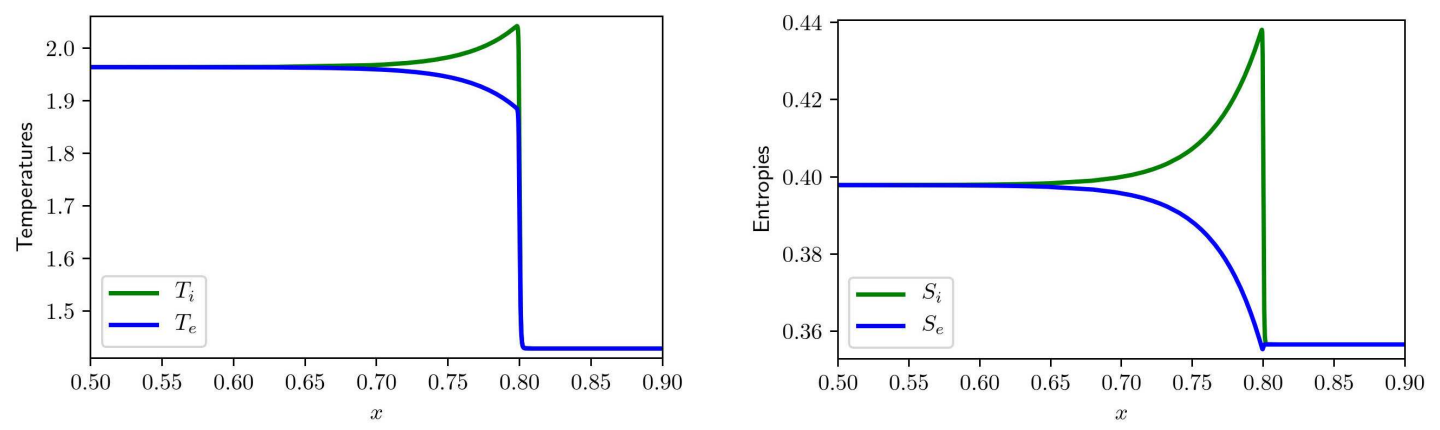

Figure 5: Temperature (left) and entropy (right) for the piston shock wave test case at time 0.4 in the case $m_{i} / m_{e}=2000$.

\subsubsection{Mono-temperature Sedov test case}

We now consider the Sedov problem [15], and we choose a diatomic perfect gas $(\gamma=1.4)$ characterized by its heat capacity $C_{v}=1$. At the initial time, we use a uniform density $\rho(t=$ $0)=1$, a null velocity $\boldsymbol{u}(t=0)=\mathbf{0}$ and we set a delta-function energy source prescribing the pressure in the cell containing the origin as follows

$$
p_{\mathrm{or}}^{e}(t=0)=(\gamma-1) \rho(t=0) \frac{\varepsilon_{\mathrm{or}}^{e}}{v_{\mathrm{or}}}
$$

where $v_{\text {or }}$ denotes the volume of the cell that contains the origin and $\varepsilon_{\text {or }}^{e}$ is the total amount of released energy. By choosing $\varepsilon_{\text {or }}^{e}=0.244816$, as it is suggested in [15], the solution consists in a diverging shock whose front is located at radius $r=\sqrt{x^{2}+y^{2}}=1$ at time $t=1$. The numerical domain used is a square $[0,1.2]^{2}$ discretized with 50 cells on each direction. As the test case is a reduction of a cylindrical physical phenomenon in a 2D plane, we impose a symmetry boundary condition on the left and on the bottom. On the right and on the top we impose a null velocity. The time step is chosen according to a CFL number condition equal to 0.45 . The final time is 1 . Fig. 6 shows the density field 


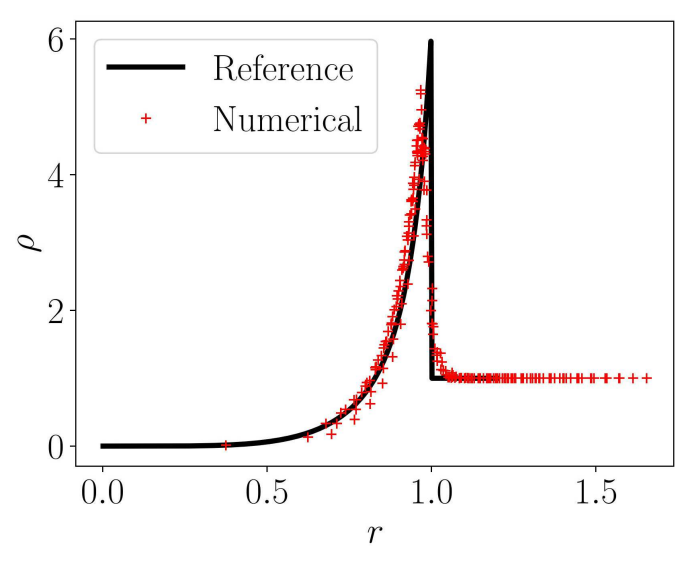

(a) Density field with $20 \times 20$ cells

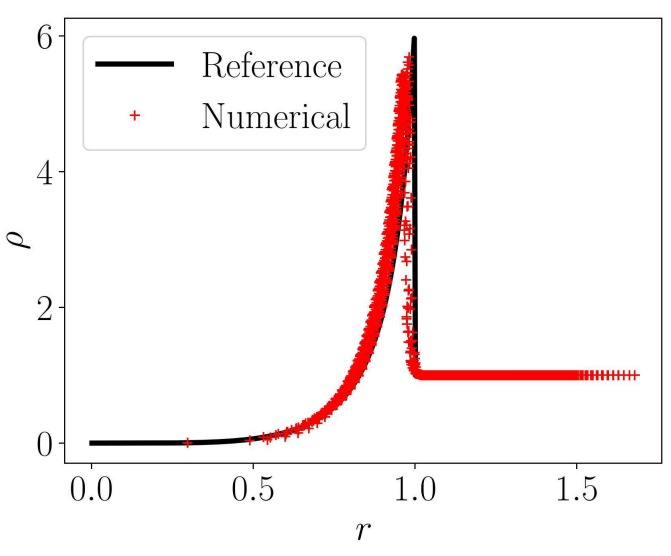

(b) Density field with $50 \times 50$ cells

Figure 6: Density field for the Sedov test case at time $t=0.05$.
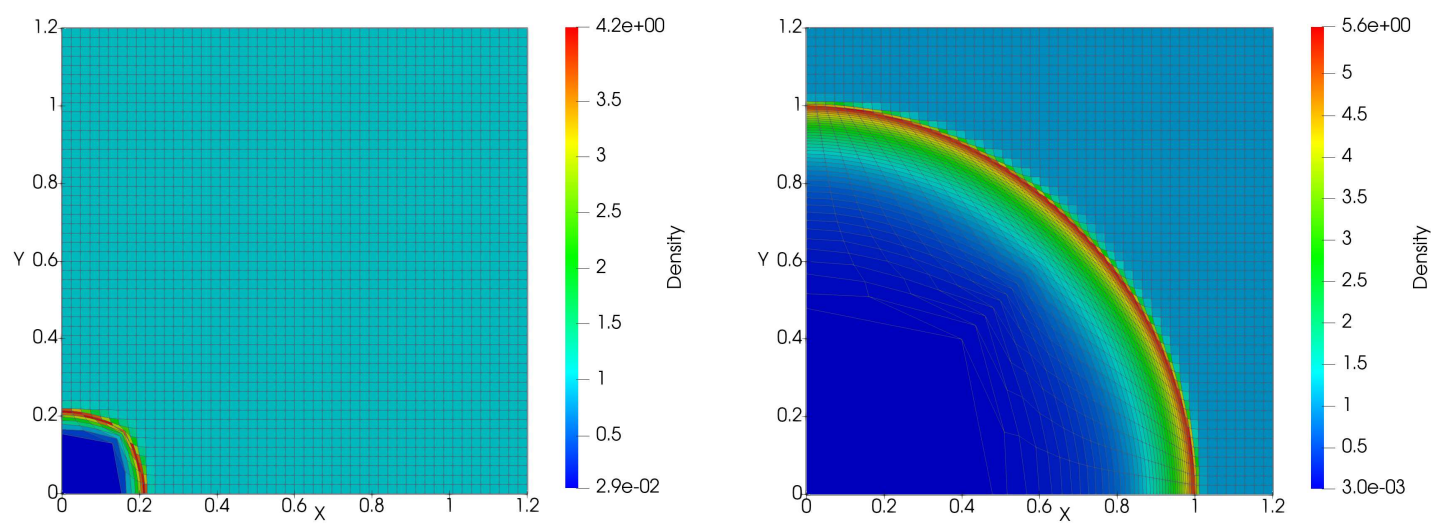

Figure 7: Density field for the Sedov test case ( $t=0.05$ on the left, $t=1$ on the right).

as a function of the radius $r$ and Fig. 7 displays the solution at times $t=0.05$ and $t=1$. According to Figs. 6 and 7, the scheme preserves the flow symmetries and ensures the convergence. In spite of a small dispersion of the values before the shock, all waves are correctly captured.

\subsubsection{Bi-temperature Sedov test case}

As described in the previous sections, we consider a perfect diatomic gas. The heat capacity of ions and electrons are taken equal to 1 . In order to examine the capability of the scheme to simulate a bi-temperature problem we adapt the previous Sedov test case using a mass ratio of $1 / 2000$. The initial energy is distributed only on electrons and computed according to formula (3.2). The coupling coefficient $\kappa$ is constant and taken equal to 0.01 . Fig. 8 shows the sum of electronic and ionic density. By definition, this sum has 


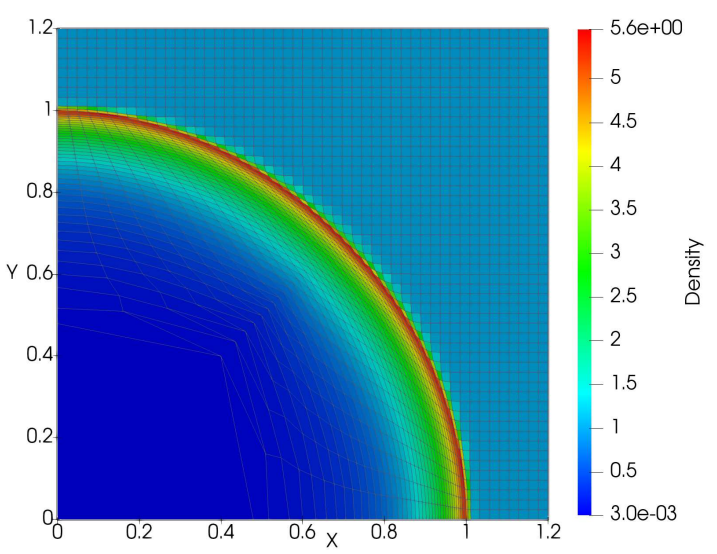

Figure 8: Density for the Sedov bi-temperature test case at $t=1$.
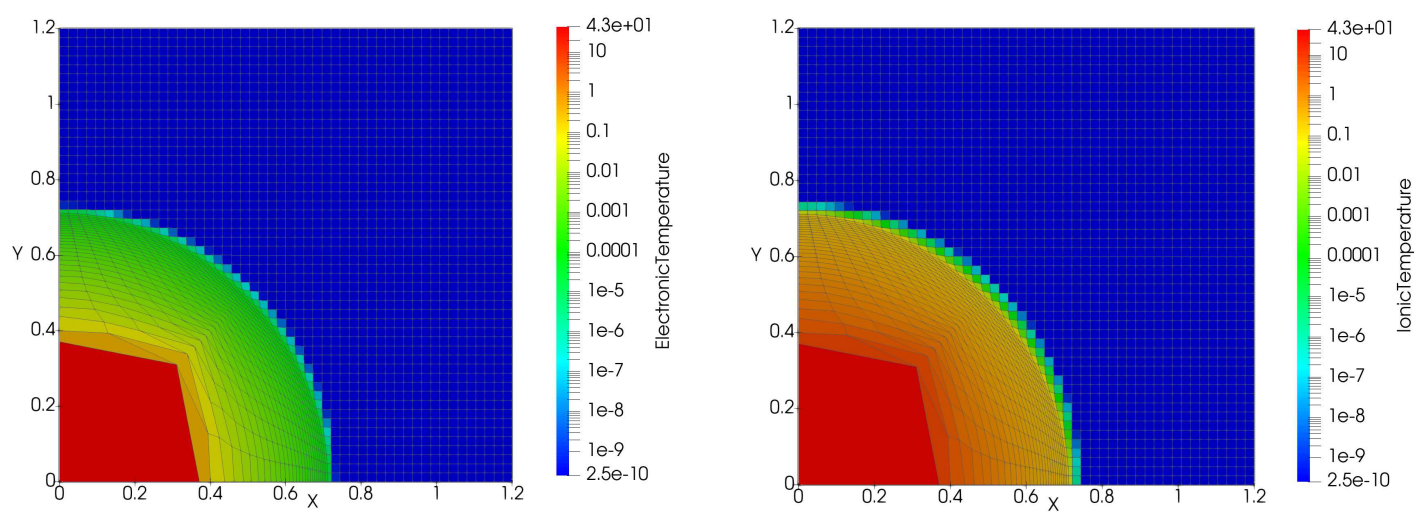

Figure 9: Electronic and ionic temperatures for the Sedov bi-temperature test case with initial energy on electrons at time $t=0.5$.

to be equal to the density field shown on Fig. 7. Graphically, we can observe that both density fields are the same. In order to illustrate the coupling effects, we may observe the evolution of the temperature profiles. Figs. 9 and 10 report the evolution of ionic and electronic temperatures at different times. The ions being heavier than the electrons, the shock process dissipates more entropy on ions, consequently ionic temperature is higher than electronic temperature at the end of the computation. After the shock, thanks to the relaxation process, we observe a coupling between temperatures. We choose to put all the initial internal energy on electrons in order to assess the stability of the scheme. Indeed, since there is almost no numerical production on the electron energy equation, one could expect stability issues. However, it is observed the scheme always remains perfectly stable. 

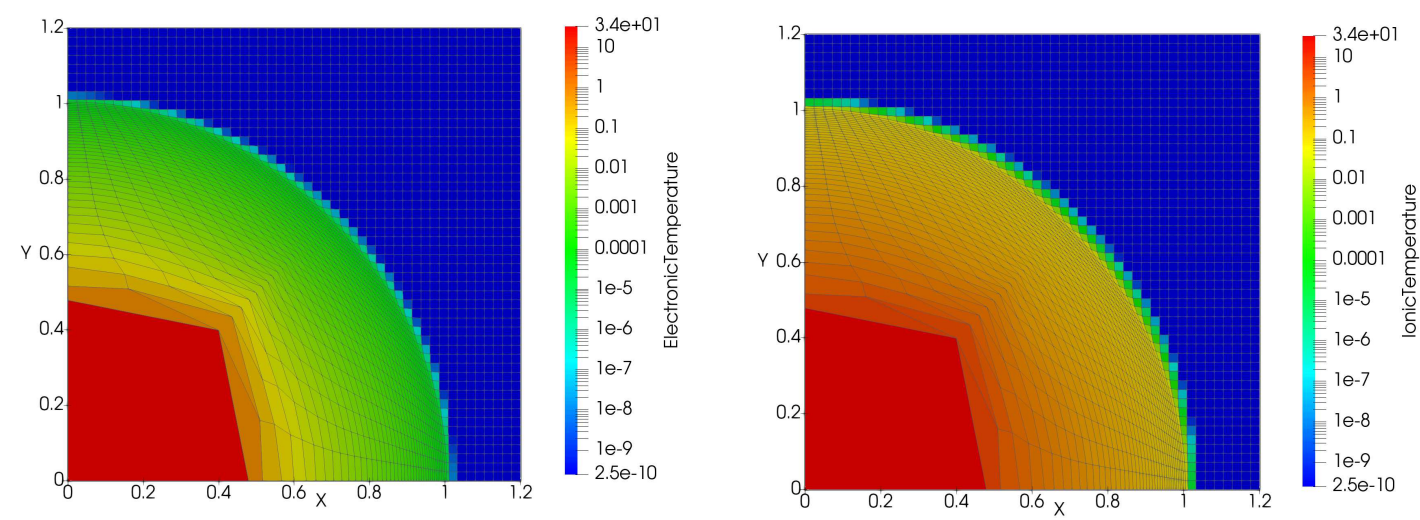

Figure 10: Electronic and ionic temperatures for the Sedov bi-temperature test case with initial energy on electrons at time $t=1$.

\subsection{Saltzmann test case}

\subsubsection{Mono-temperature Saltzmann test case}

The domain is filled with a mono-atomic gas whose initial configuration is $(\rho, p, u)=$ $\left(1,10^{-10}, \mathbf{0}\right)$. The compression is computed by applying the velocity condition on the left boundary. On all other boundaries, we impose a symmetry condition. The exact solution is a planar shock wave that moves from left to right. The final time is $t=0.7$. Figs. 11 and 12 respectively show the density field and its convergence. The scheme preserves the onedimensional solution very well. On the other hand we can observe that the numerical overshoot on the head of the wave decreases while the number of cells increases.

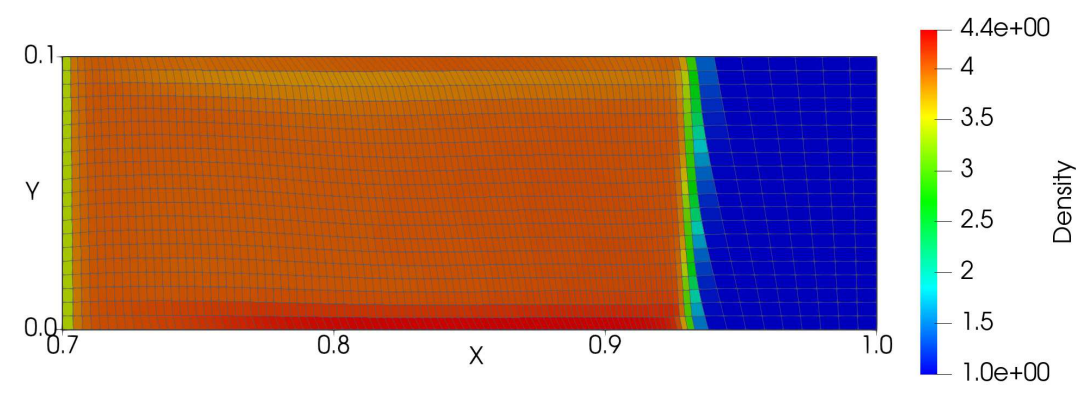

Figure 11: Density field for the Saltzmann test case at time $t=0.7$.

\subsubsection{Bi-temperature Saltzmann case test}

We use the configuration of the Saltzmann mono-temperature case but now set $\kappa=20$. We choose to put all the initial internal energy on electrons in order to assess the stability of the scheme. Indeed, since there is almost no numerical dissipation on the electron energy equation, one could expect stability issues. However, as it is observed in Fig. 13, where 


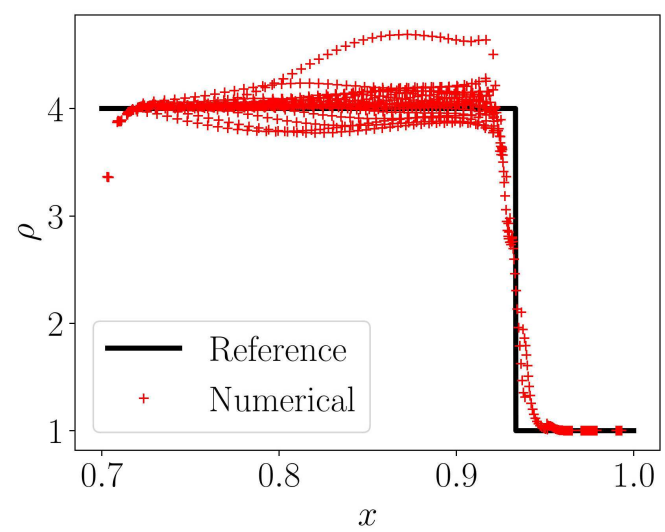

(a) Density field with $50 \times 20$ cells

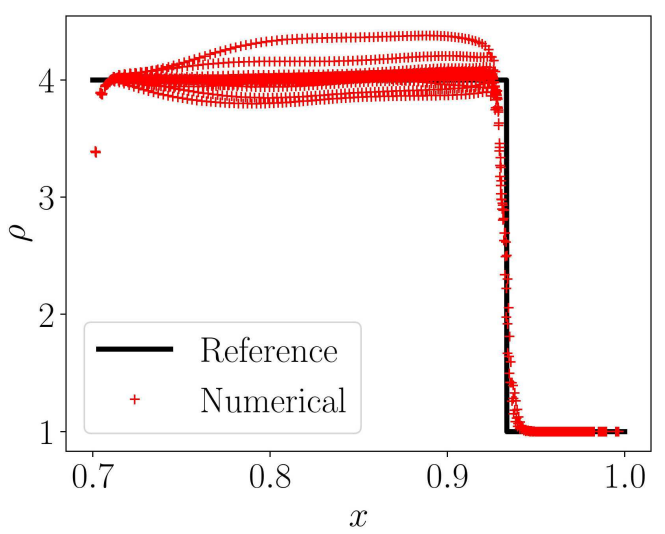

(b) Density field with $100 \times 20$ cells

Figure 12: Density for the Saltzmann test case at time $t=0.7$.
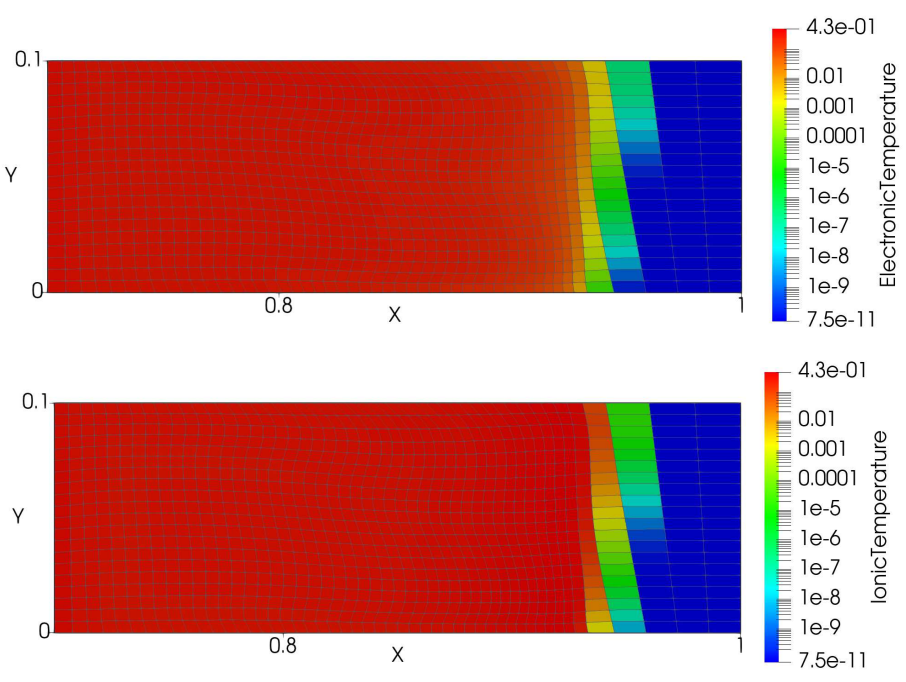

Figure 13: Electronic and ionic temperatures for the Saltzmann bi-temperature test case with initial energy on electrons at time $t=0.7$.

the electronic and ionic temperature profiles are displayed at time $t=0.7$, the scheme is perfectly stable and the one-dimension structure of the solution is preserved.

\subsection{Radiation test cases}

\subsection{1 $0 \mathrm{D}$ test case}

We start by studying the relaxation process. To do so, four problems taken from [8] and [9] are reproduced. For the first three problems, we set a source term $Q$ on ions 
Table 1: Parameters and initial quantities used for OD problems.

\begin{tabular}{||c|c|c|c|c||}
\hline & Problem 1 & Problem 2 & Problem 3 & Problem 4 \\
\hline$c$ & 29.979 & 29.979 & 29.979 & 29.979 \\
$a$ & 0.02372 & 0.02372 & 0.02372 & 0.02372 \\
$\sigma_{P}$ & $0.5 T_{e}^{-2}$ & $0.1 T_{e}^{-2}$ & $0.5 T_{e}^{-2}$ & $0.1 T_{e}^{-2}$ \\
$\kappa$ & 0.1 & $0.01379 T_{e}^{-0.5}$ & 0.1 & $0.01379 T_{e}^{-0.5}$ \\
$C_{v, i}$ & 0.15 & 0.15 & 0.15 & 0.15 \\
$C_{v, e}$ & 0.3 & $0.3 T_{e}$ & 0.3 & $0.3 T_{e}$ \\
$T_{i}(t=0)$ & $2.52487 \cdot 10^{-5}$ & $2.52487 \cdot 10^{-5}$ & $2.52487 \cdot 10^{-1}$ & $2.52487 \cdot 10^{-5}$ \\
$T_{e}(t=0)$ & $2.52487 \cdot 10^{-5}$ & $2.52487 \cdot 10^{-5}$ & $2.52487 \cdot 10^{1}$ & $2.52487 \cdot 10^{-5}$ \\
$T_{r}(t=0)$ & $2.52487 \cdot 10^{-5}$ & $2.52487 \cdot 10^{-5}$ & $2.52487 \cdot 10^{-1}$ & $2.52487 \cdot 10^{-5}$ \\
$A$ & 75.19884 & 15.03978 & 75.19884 & 15.03978 \\
\hline
\end{tabular}
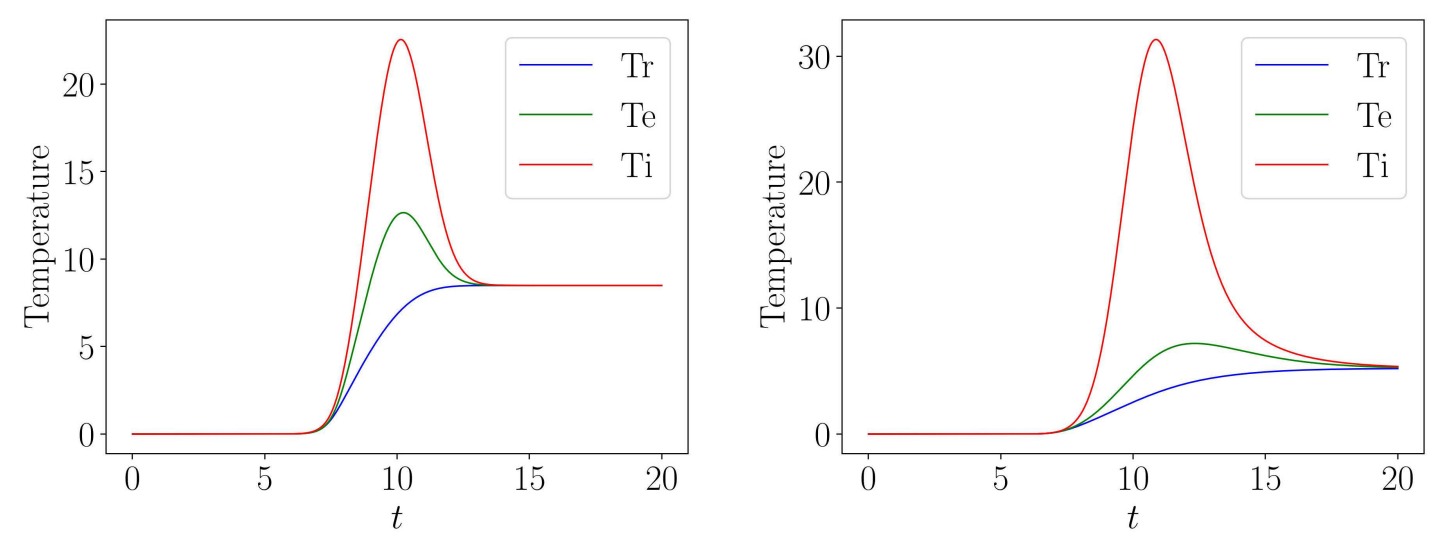

Figure 14: Temperatures for $0 \mathrm{D}$ case test (problem 1 on left, problem 2 on right).

such that

$$
\int_{t_{1}}^{t_{2}} Q \mathrm{~d} t=\frac{A}{2}\left(\operatorname{erf}\left(\frac{t_{2}-t_{c}}{\sqrt{2} t_{w}}\right)-\operatorname{erf}\left(\frac{t_{1}-t_{c}}{\sqrt{2} t_{w}}\right)\right)
$$

where $t_{c}=10, t_{w}=1$ and erf is the standard error function. For the last problem the source term is moved on photons. Table 1 summarizes the general parameters. The final time is $t=20$. Fig. 14 shows temperatures for problems 1 and 2. After a short steady state, we observe a decoupling of the temperatures. Due to the source term, the ionic temperature is higher than others, the numerical scheme gives correct results according to [8]. Fig. 15 reports the time evolution of temperatures for problems 3 and 4. Graphically, the results presented in [8] are recovered. In particular, we denote, thanks to the convex combinations, the robustness of the scheme to ensure the temperature positiveness even in a stiffness context as the initial time of problem 3 for instance. 

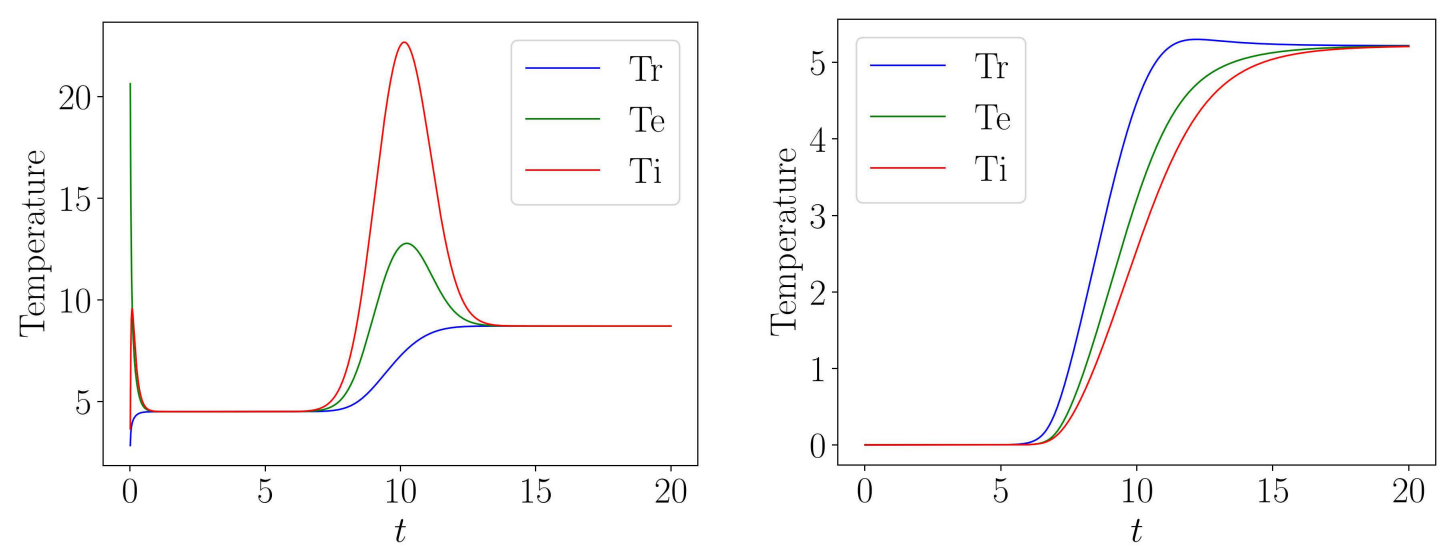

Figure 15: Temperatures for $0 \mathrm{D}$ case test (problem 3 on left, problem 4 on right).

\subsubsection{Marshak wave without and with electron and ion conductions}

We consider a Marshak problem [32] in a one dimension domain. We set hydrodynamic variables to $\rho=1$ and $\boldsymbol{u}=\mathbf{0}$. The speed of light $c$ is taken equal to 299.79 and the radiation constant $a$ is equal to 0.01372 . We set the coupling coefficient $\kappa$ at a very large value $\left(\kappa=10^{30}\right)$ to ensure a unique matter temperature $T_{m}$ such that $T_{m}=T_{e}=T_{i}$. The Rosseland and Planck opacities are such that $\sigma_{P}=\sigma_{R}=300 / T_{m}^{3}$ and the heat capacities such that $C_{v, i}=0.27$ and $C_{v, e}=0.03$. At the initial time, the temperatures $T_{m}$ and $T_{r}$ are equal to $10^{-6}$; we impose a temperature $T_{m}=T_{r}=1$ on the left boundary of the domain and Neumann outgoing flux condition on the right. We work on a domain $[0,0.5]$ discretized with 500 cells. A constant time step equal to $10^{-3}$ is used. As the exact solution of this problem is unknown, we use a refined solution computed with a reference one dimension code and report the temperature on Fig. 16 for several final times. This numerical test case has also been performed by taking into account electron and ion Spitzer-Harm conductivities of the form

$$
F_{\alpha}=K T_{\alpha}^{5 / 2} \nabla T_{\alpha}, \quad \alpha=e, i .
$$

Recall here that these terms are treated with a standard operator splitting strategy. The numerical results are displayed in Fig. 17 in the case $K=0.1$ (left) and $K=1$ (right). It is observed that adding the ion and electron conduction terms impacts the wave propagation speed. The numerical solutions match perfectly with the reference solutions. In addition, we recall that the coupling to matter (here to electrons and ions) makes the problem strongly nonlinear. Since no operator splitting strategy is used here, an assessment of the number of the non-linear solver iterations for different regimes of coupling may be studied. In Fig. 18, for the Marshak wave problem without electron and ion conductions, the number of iterations has been displayed with respect to time for different Planck opacities, i.e for different regimes of coupling. It is observed that the number of iterations decreases as the coupling between radiation and matter becomes less stiff. 

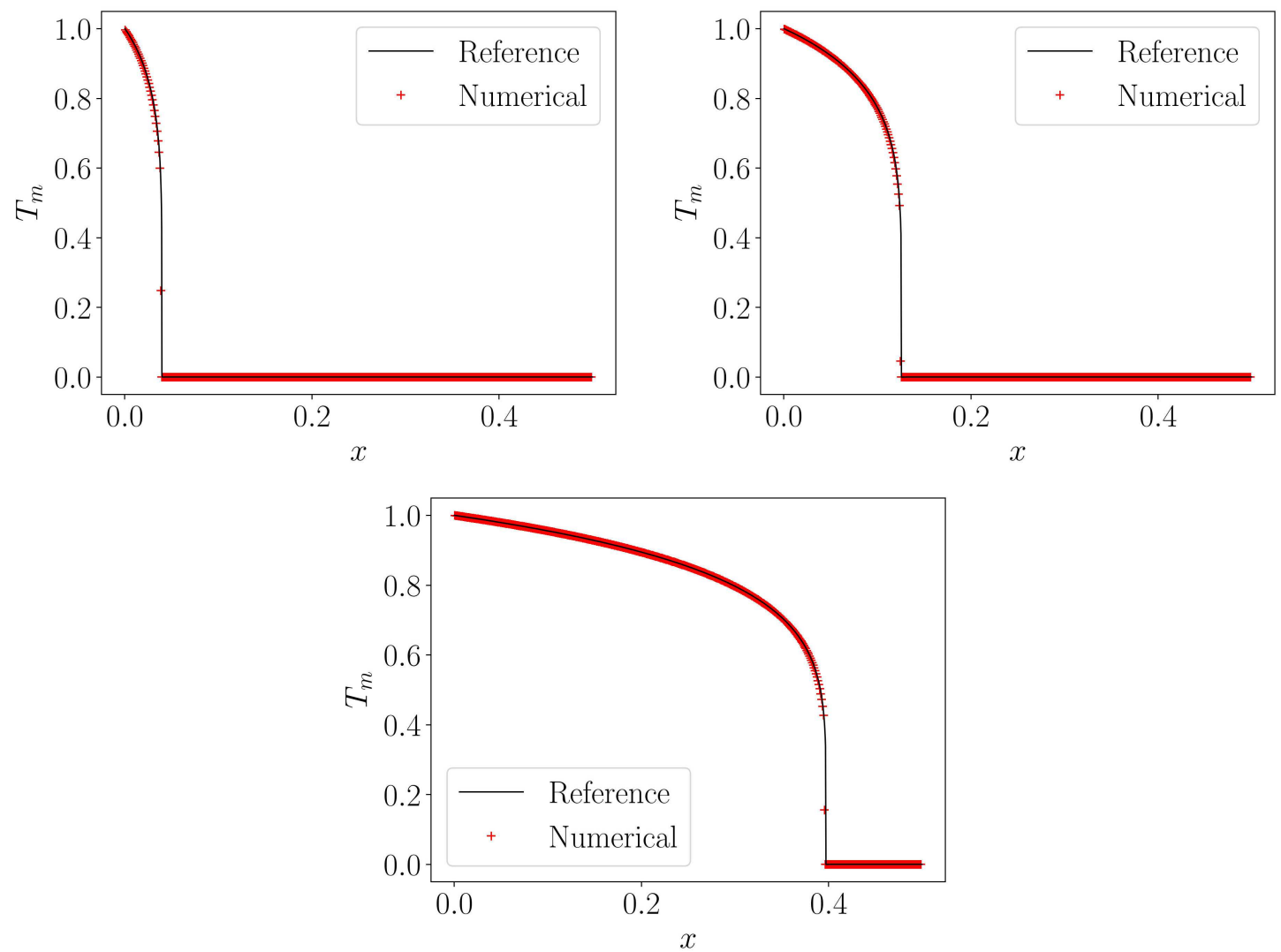

Figure 16: Temperature profiles for Marshak wave (at time 0.074 on left, at time 0.74 on right and at time 7.4).
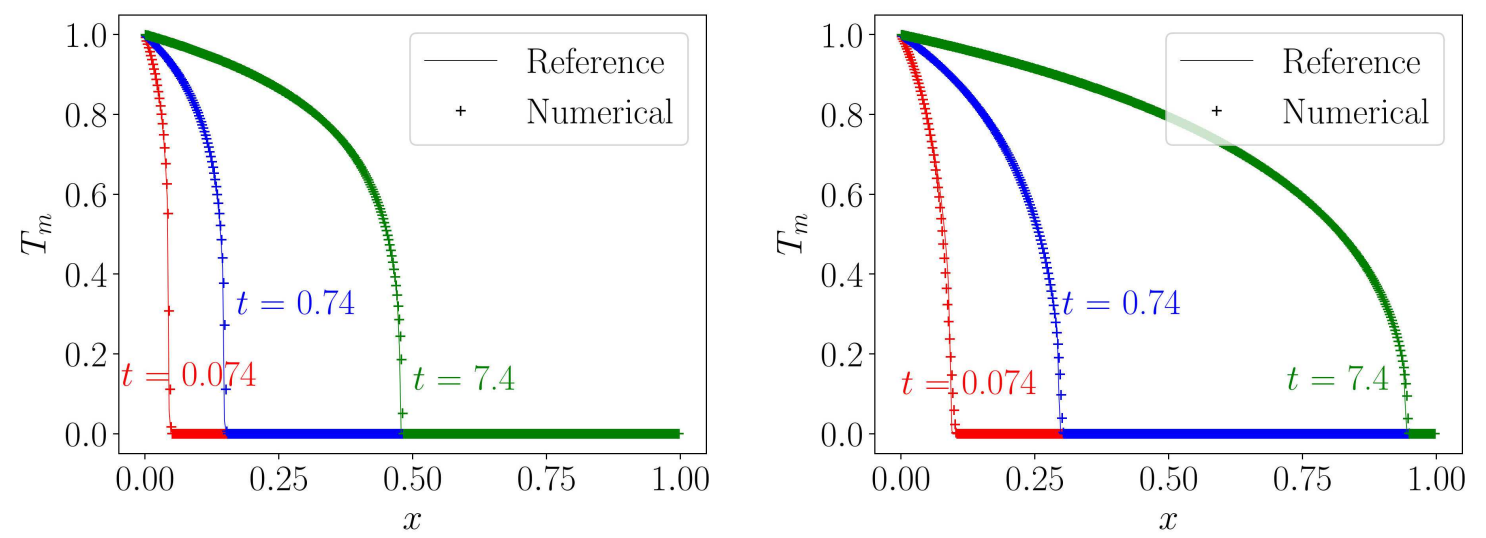

Figure 17: Temperature profiles for Marshak wave with electron and ion conductivities. 


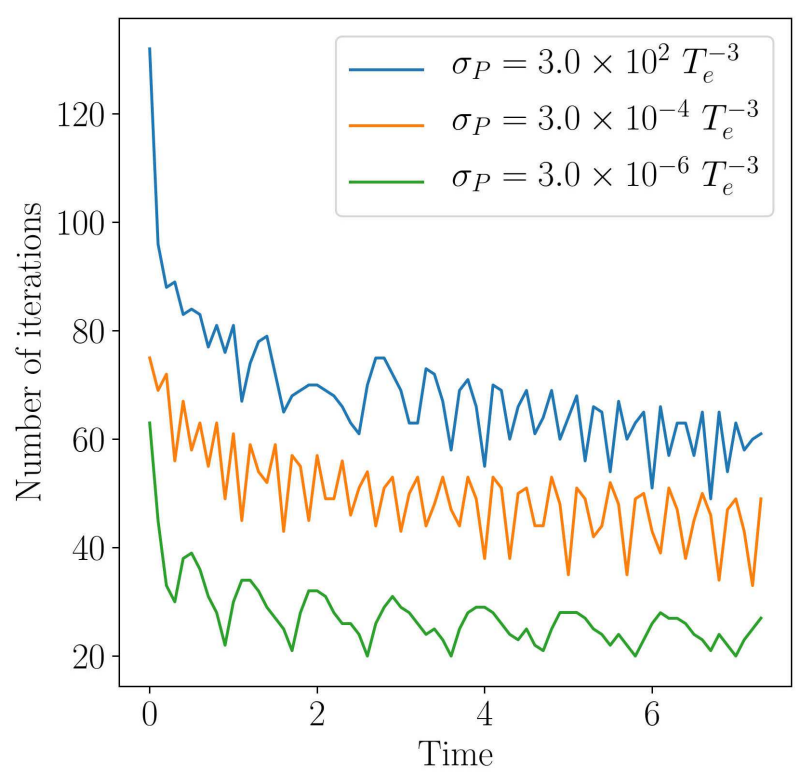

Figure 18: Number of non-linear solver iterations for the Marshak wave problem with respect to time for different Planck opacities (different coupling regimes).

\subsection{Radiation hydrodynamics test cases}

\subsubsection{Shock tube problem in strong equilibrium regime}

The first case coupling hydrodynamics and radiation phenomena is a shock tube problem in the strong equilibrium regime [35]. The speed of light $c$ and the radiation constant $a$ are fixed to $2.9979 \cdot 10^{10}$ and $7.5657 \cdot 10^{-15}$ respectively and we consider an ideal atomic gas $\left(\gamma=\frac{5}{3}\right)$ of heat capacity $C_{v}$ such that

$$
C_{v}=\frac{k}{(\gamma-1) \mu m_{H}}
$$

where $k$ is the Boltzmann constant taken equal to $1.380649 \cdot 10^{-16}, \mu$ is the mean molecular weight equal to 1 and $m_{H}$ is the mass of a hydrogen atom equal to $1.6733 \cdot 10^{-24}$. The Planck and Rosseland opacities are respectively chosen as follows

$$
\sigma_{P}=10^{8}, \quad \sigma_{R}=10^{6}
$$

As in the Marshak wave test case, in order to ensure a unique matter temperature, we set the parameter $\kappa$ to $10^{30}$. We work on the domain $[0,100]$, at the initial time the radiation is in equilibrium with the matter and we set a Riemann problem whose discontinuity is 

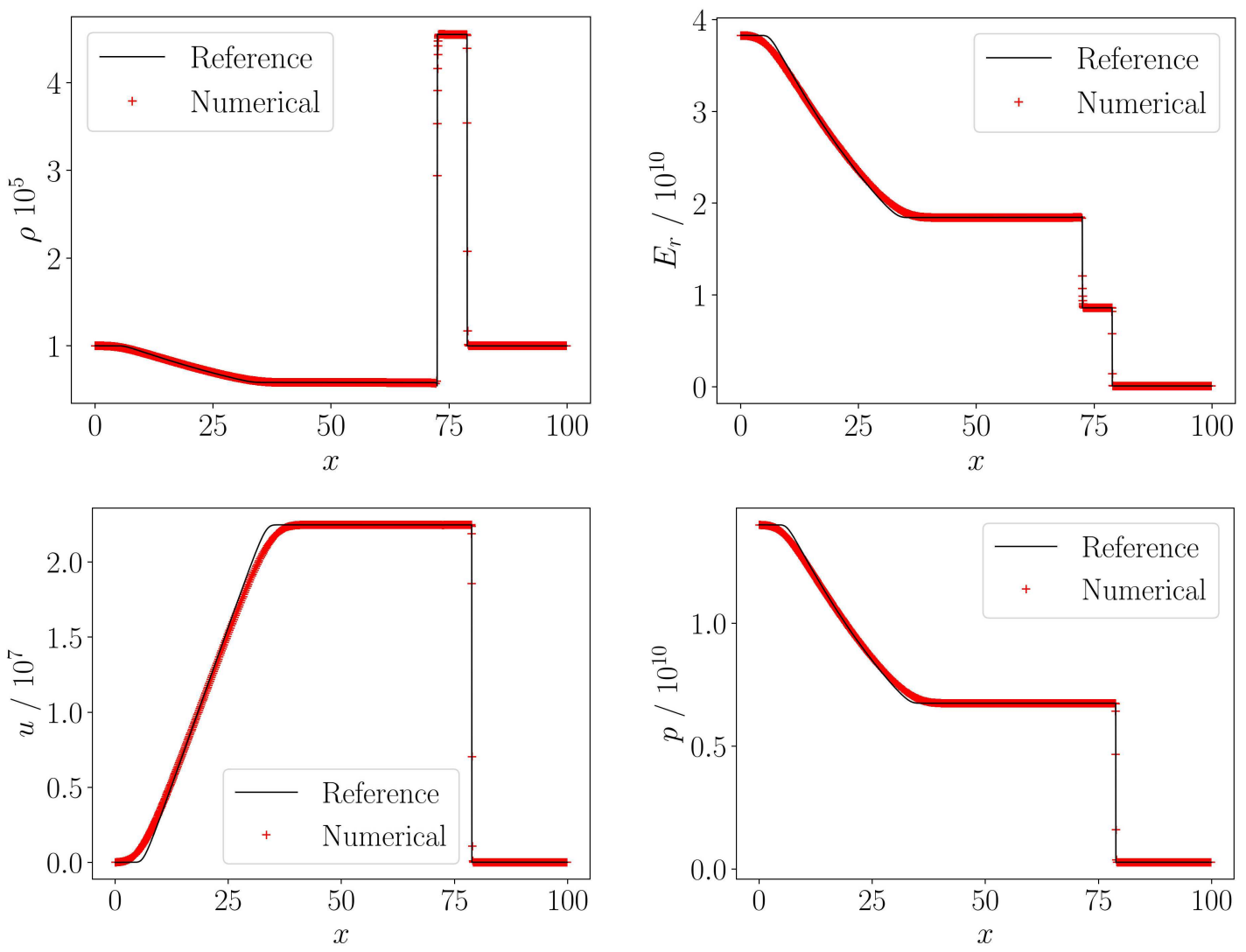

Figure 19: Results for shock tube problem at time $t=10^{-6}$.

located at $x=50$ such that

$$
\left(\begin{array}{c}
\rho \\
\boldsymbol{u} \\
T_{r} \\
T_{m}
\end{array}\right)(t=0)=\left(\begin{array}{c}
10^{-5} \\
\mathbf{0} \\
1.5 \cdot 10^{6} \mathbf{1}_{x \leq 50}+3 \cdot 10^{5} \mathbf{1}_{x>50} \\
1.5 \cdot 10^{6} \mathbf{1}_{x \leq 50}+3 \cdot 10^{5} \mathbf{1}_{x>50}
\end{array}\right) .
$$

Using (3.3), (3.4) and (3.5), it can be shown that the problem is nearly governed by the Euler equations with the equation of state $p=(\gamma-1) \rho C_{v} T+\frac{a T^{4}}{3}$. Consequently, we may compute a reference solution with a mono temperature code. We discretize the domain with 800 cells and we use a time step computed according to a CFL number equal to 0.45 . Fig. 19 shows the physical quantities for a final time $t=10^{-6}$. The numerical scheme produces results that agrees with the reference solution in shock and rarefaction waves.

\subsubsection{Radiation shock wave}

The second case test implicating a coupling between hydrodynamics and radiation phenomena consists in the study of a stationary radiative shock [18]. Actually, radiation may 
completely modify the structure of a shock due to diffusion and interactions with matter. In this case, the pre-shock matter is firstly heated by diffused radiation; then an embedded hydrodynamic shock produces a brutal raise of the temperature. Finally, because of coupling coefficients, the temperature cools down to the radiation temperature in a relaxation region. In order to illustrate the capability of the scheme to ensure a correct capture of this shock structure, we first study a sub-critical shock and we use the parameters of [35]. As in previous sections, the speed of light $c$ and the radiation constant $a$ are fixed to $2.9979 \cdot 10^{10}$ and $7.5657 \cdot 10^{-15}$ respectively and we consider an ideal atomic gas $\left(\gamma=\frac{5}{3}\right)$ whose heat capacity is given by Eq. (3.3). We work on $[0,2000]$ and the quantities are initialized as follows

$$
\left(\begin{array}{c}
\rho \\
u \\
T_{r} \\
T_{m}
\end{array}\right)=\left(\begin{array}{c}
5.45887 \cdot 10^{-13} \\
\left(2.35435 \cdot 10^{5}, 0\right)^{T} \\
100 \\
100
\end{array}\right) \mathbf{1}_{x \leq 1500}+\left(\begin{array}{c}
1.24794 \cdot 10^{-12} \\
\left(1.02987 \cdot 10^{5}, 0\right)^{T} \\
207.757 \\
207.757
\end{array}\right) \mathbf{1}_{x>1500} .
$$

The Rosseland and Planck opacity are taken equal to 0.848902 and $3.92664 \cdot 10^{-5}$ respectively. From a numerical point of view, the domain is discretized with 1000 cells and the time step is computed with a CFL number equal to 0.49 . Reference solutions are computed from a semi analytical solution given by the procedure of [18]. We report on Fig. 20 the density field, the radiation and matter temperatures at time $t=0.05$. Here, since a stationary solution is expected [18], the numerical simulation is performed until a stationary state is reached. In order to compare the results with the semi analytical solution, we proceed with a slight shifting of the numerical solution to ensure the position of the shock at $x=1500[5,35]$. The numerical results are in very good agreement with the reference solutions in the precursor region as well as in the relaxation region. In particular, it is observed that the temperature spike is recovered with a very good accuracy.

The previous test case is a sub-critical shock wave because the maximal temperature of the precursor region is smaller than the right temperature. Another possible test case is a super-critical shock wave. In this case the maximal temperature of the precursor region is equal to the right temperature. In order to simulate this type of shock, we use, as previously, the parameters of [35]. We work on [0,5000], the quantities are initialized as follows

$$
\left(\begin{array}{c}
\rho \\
u \\
T_{r} \\
T_{m}
\end{array}\right)(t=0)=\left(\begin{array}{c}
5.45887 \cdot 10^{-13} \\
\left(5.885885 \cdot 10^{5}, 0\right)^{T} \\
100 \\
100
\end{array}\right) \mathbf{1}_{x \leq 3500}+\left(\begin{array}{c}
1.24794 \cdot 10^{-12} \\
\left(1.63592 \cdot 10^{5}, 0\right)^{T} \\
855.72 \\
855.72
\end{array}\right) \mathbf{1}_{x>3500}
$$

The space domain is discretized with 2500 cells and the time step is chosen with a CFL number equal to 0.49 . As previously, the reference solution is computed with the procedure of [18]. Fig. 21 shows the numerical results at time $t=0.04$. As in the sub-critical case, the numerical results are very satisfying. In particular the ZelDovich peak [34] is captured with a good accuracy. 

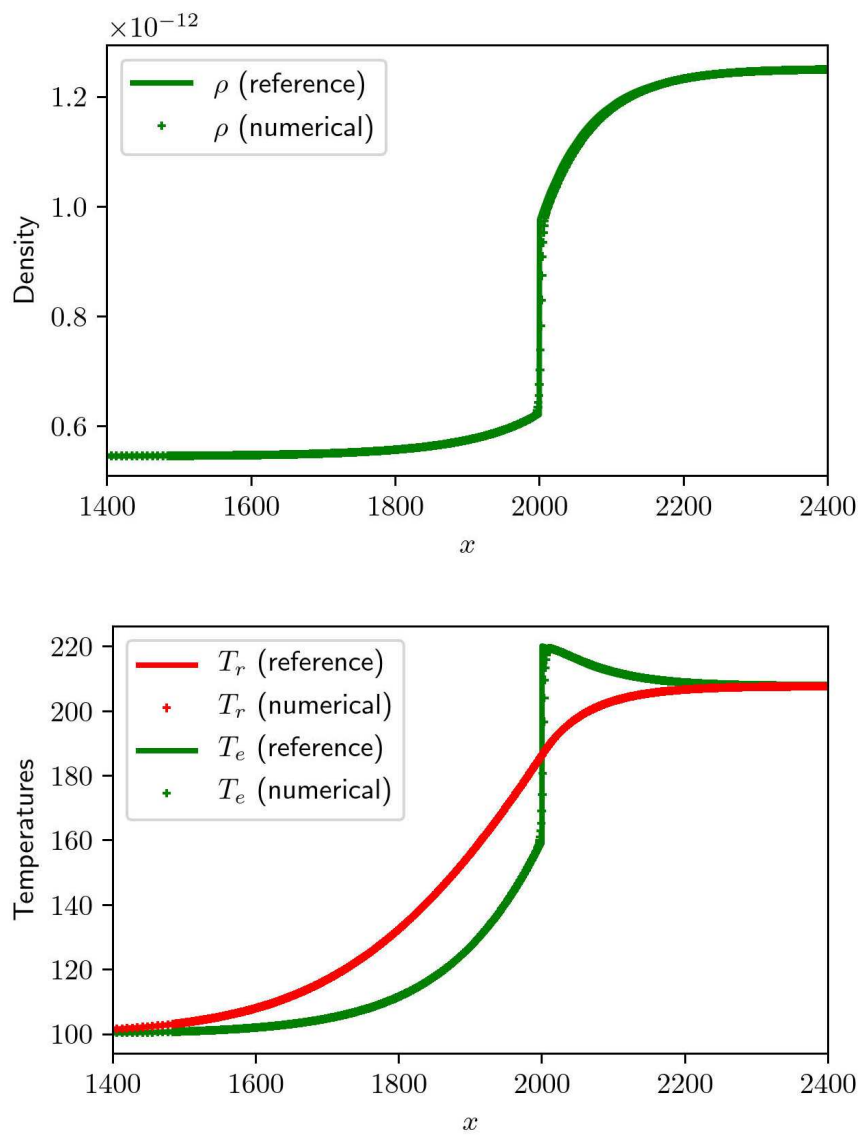

Figure 20: Density and temperature fields for the sub-critical test case at time 0.05 .

\subsubsection{Radiative blast wave}

In order to test the performance of the scheme in a two dimensional space, we adapt the test case of [35] to a two dimensional plan. As previously, the speed of light $c$ and the radiation constant $a$ are fixed to $2.9979 \cdot 10^{10}$ and $7.5657 \cdot 10^{-15}$ respectively and we consider an ideal atomic gas $\left(\gamma=\frac{5}{3}\right)$ whose heat capacity is given by Eq. (3.3). Since the problem has a cylindrical symmetry, we work on the square $\left[0,10^{14}\right]^{2}$ and we use symmetry conditions on the left and on the lower boundaries. Initially, the matter verifies $\rho(t=0)=5 \cdot 10^{-6}, \boldsymbol{u}(t=0)=\mathbf{0}$, the radiation is at equilibrium with matter and we set a high temperature in a circle centered around the origin

$$
T_{m}(t=0)=T_{r}(t=0)=10^{7} \mathbf{1}_{r<2 \cdot 10^{12}}+10^{3} \mathbf{1}_{r \geq 2 \cdot 10^{12}} .
$$

The Rosseland and Planck opacities are taken equal to $2 \cdot 10^{-10}$ and $2 \cdot 10^{-16}$ respectively. We discretize the spatial domain with 100 cells on each direction and we use a time step computed according to a CFL number of 0.49 . Numerical results are summarized on 

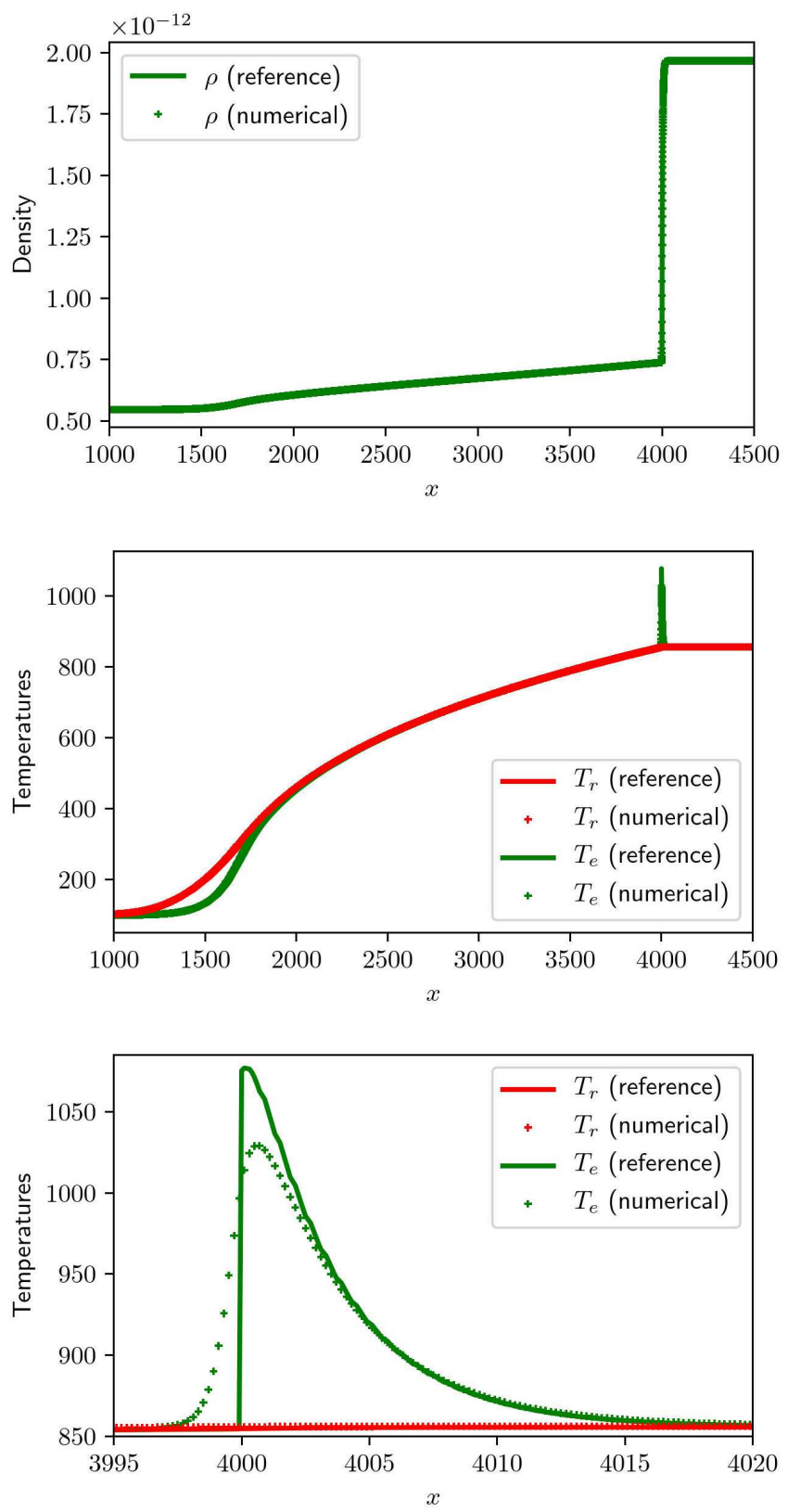

Figure 21: Density and temperature fields for the super-critical test case at time 0.4 .

Figs. 22 to 24 . We denote a very good wave symmetry conservation. The geometry of the wave and its maximal values are very close to the ones of the wave introduced on axisymmetric problem in [35]. 

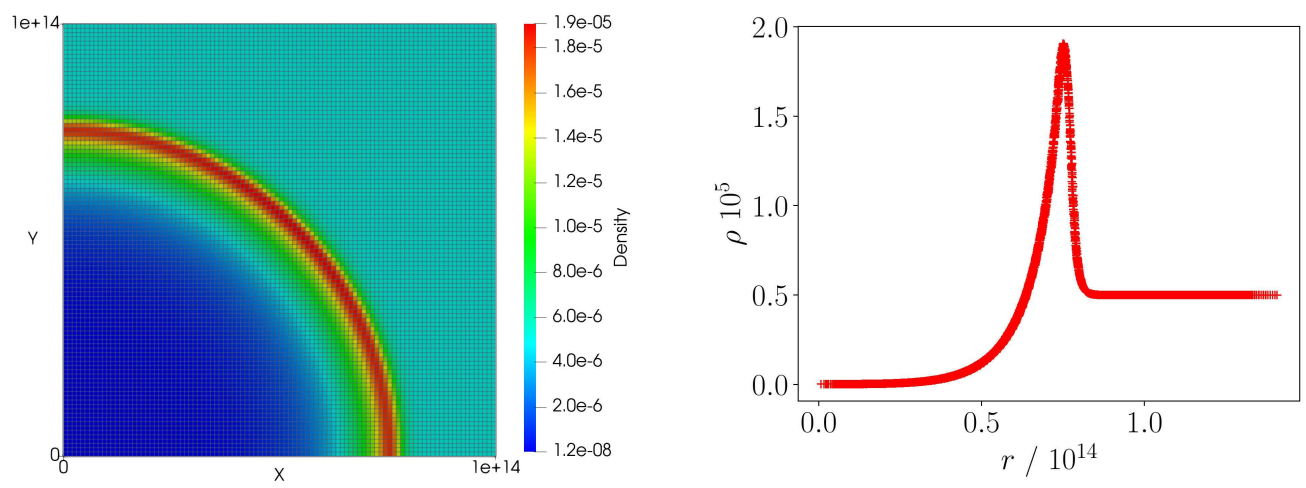

Figure 22: Density field at time $5 \cdot 10^{5}$.
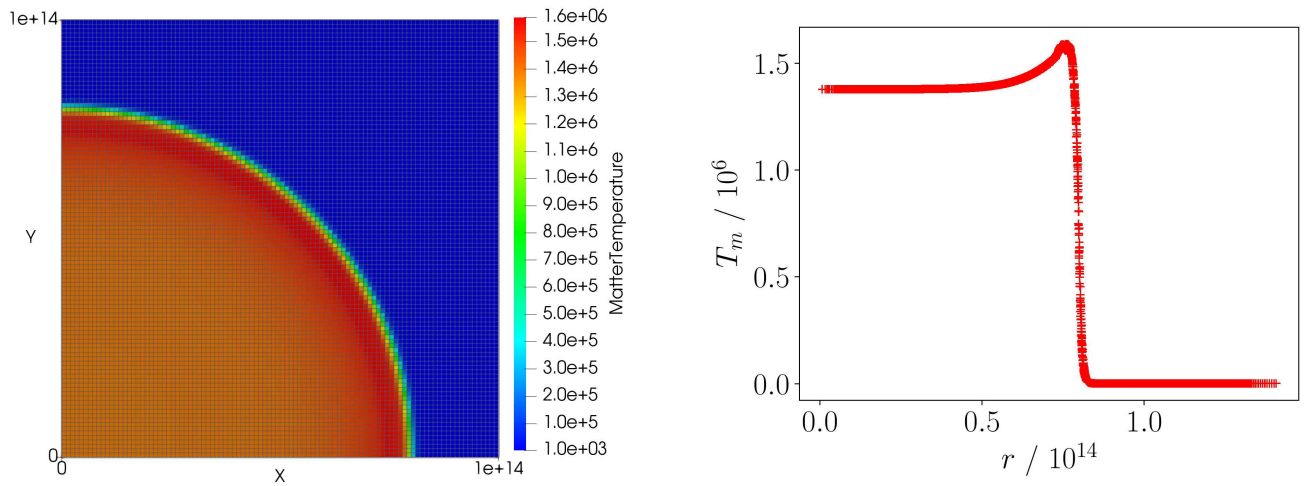

Figure 23: Matter temperature field at time $5 \cdot 10^{5}$.
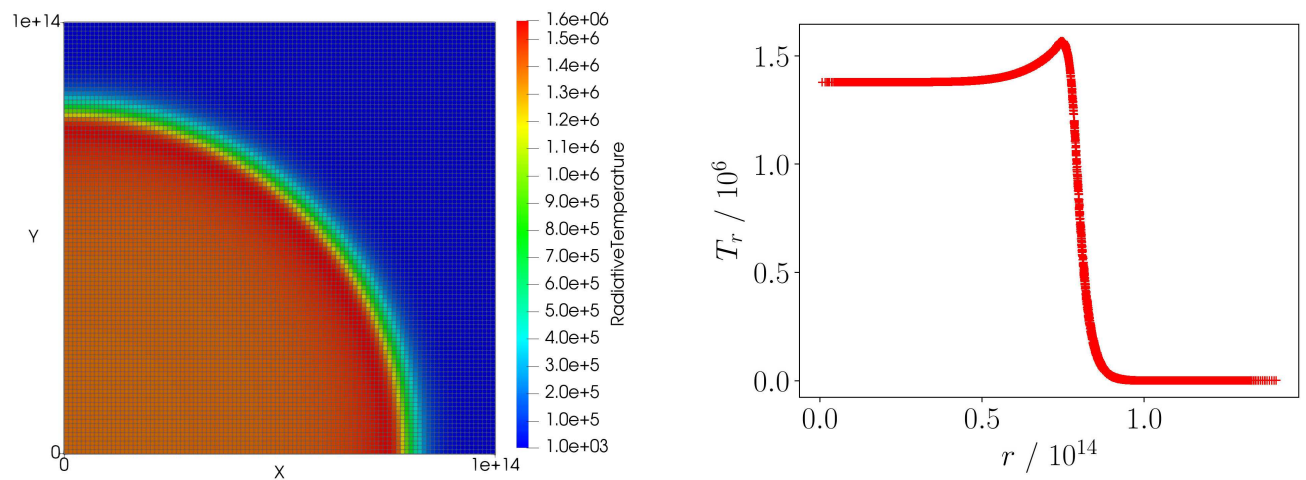

Figure 24: Radiative temperature field at time $5 \cdot 10^{5}$. 


\section{Conclusion}

A positivity-preserving and entropy dissipative (per species) Eucclhyd-like scheme has been presented for three-temperature grey diffusion radiation hydrodynamics. To achieve the correct entropy production for each species we choose to work with internal energy quantities (the global scheme remains conservative in energy) and the dissipation matrices are chosen in order to enforce a discrete entropy production as prescribed in [34]. Then, the positivity-preserving property of all temperatures is obtained thanks to a reformulation of the model which enables the derivation of a convex-combination based scheme. The iterative procedure leads to the resolution of a linear set of equations associated to a M-matrix and a positive right-hand side. Pure hydrodynamics, pure radiation and radiation-hydrodynamics numerical tests demonstrate the claimed properties at the discrete level. In addition, the robustness of the studied numerical strategy has been observed as well as its efficiency (even setting all the internal energy on electrons) even if the number of subiterations increases as the coupling between radiation and matter becomes stiff. As natural perspectives, we intend to extend the present numerical strategy to include multi-material aspects and mixtures.

\section{Appendix}

In this section the proofs of the semi-discrete entropy production and discrete total energy conservation are detailed.

Property A.1 (Semi-discrete entropy production). The total semi-discrete entropy in the domain is dissipated

$$
\sum_{c} m_{c} \frac{\mathrm{d} \eta_{c}}{\mathrm{~d} t} \geq 0
$$

Proof. Using definitions (1.7) and (1.8), the discrete entropy evolution equations read

$$
\left\{\begin{aligned}
m_{c} T_{r, c} \frac{\mathrm{d} \eta_{c}^{r}}{\mathrm{~d} t}= & \sum_{p \in \mathcal{P}(c)} \boldsymbol{M}_{p c}^{r}\left(\boldsymbol{u}_{p}-\boldsymbol{u}_{c}\right) \cdot\left(\boldsymbol{u}_{p}-\boldsymbol{u}_{c}\right)+\sum_{d \in \mathcal{N}(c)} \frac{c L_{d c}}{3 \sigma_{R, d c}} \frac{E_{r, d}-E_{r, c}}{\left\|x_{c}-x_{d}\right\|_{2}} \\
& +c \sigma_{P, c} v_{\mathcal{c}}\left(a T_{e, c}^{4}-E_{r, c}\right) \\
m_{c} T_{e, c} \frac{\mathrm{d} \eta_{c}^{e}}{\mathrm{~d} t}= & \sum_{p \in \mathcal{P}(c)} \boldsymbol{M}_{p c}^{e}\left(\boldsymbol{u}_{p}-\boldsymbol{u}_{c}\right) \cdot\left(\boldsymbol{u}_{p}-\boldsymbol{u}_{c}\right)+c \sigma_{P, c} v_{c}\left(E_{r, c}-a T_{e, c}^{4}\right)+v_{c} \mathcal{K}_{c}\left(T_{i, c}-T_{e, c}\right), \\
m_{c} T_{i, c} \frac{\mathrm{d} \eta_{c}^{i}}{\mathrm{~d} t}= & \sum_{p \in \mathcal{P}(c)} \boldsymbol{M}_{p c}^{i}\left(\boldsymbol{u}_{p}-\boldsymbol{u}_{c}\right) \cdot\left(\boldsymbol{u}_{p}-\boldsymbol{u}_{c}\right)+v_{c} \mathcal{K}_{\mathcal{c}}\left(T_{e, c}-T_{i, c}\right) .
\end{aligned}\right.
$$


Consequently, the total entropy production verifies

$$
\begin{aligned}
m_{c} \frac{\mathrm{d} \eta_{c}}{\mathrm{~d} t}=m_{c} \frac{\mathrm{d} \eta_{c}^{r}}{\mathrm{~d} t}+m_{c} \frac{\mathrm{d} \eta_{c}^{e}}{\mathrm{~d} t}+m_{c} \frac{\mathrm{d} \eta_{c}^{i}}{\mathrm{~d} t} \\
=\left[\begin{array}{l}
\sum_{p \in \mathcal{P}(c)}\left(\frac{\boldsymbol{M}_{p c}^{r}}{T_{r, c}}+\frac{\boldsymbol{M}_{p c}^{e}}{T_{e, c}}+\frac{\boldsymbol{M}_{p c}^{i}}{T_{i, c}}\right)\left(\boldsymbol{u}_{p}-\boldsymbol{u}_{c}\right) \cdot\left(\boldsymbol{u}_{p}-\boldsymbol{u}_{c}\right) \\
+\frac{1}{T_{r, c}} \sum_{d \in \mathcal{N}(c)} \frac{c L_{d c}}{3 \sigma_{R, d c}} \frac{E_{r, d}-E_{r, c}}{\left\|x_{c}-x_{d}\right\|_{2}}+\frac{c \sigma_{P, c} v_{c}}{T_{r, c}}\left(a T_{e, c}^{4}-E_{r, c}\right) \\
+\frac{c \sigma_{P, c} v_{c}}{T_{e, c}}\left(E_{r, c}-a T_{e, c}^{4}\right)+\frac{v_{c} \mathcal{K}_{c}}{T_{e, c}}\left(T_{i, c}-T_{e, c}\right)+\frac{v_{c} \mathcal{K}_{c}}{T_{i, c}}\left(T_{e, c}-T_{i, c}\right)
\end{array}\right] \\
\geq\left[\begin{array}{l}
\sum_{d \in \mathcal{N}(c)} \frac{c L_{d c}}{3 \sigma_{R, d c}\left(T_{r, d}+T_{r, c}\right)} \frac{E_{r, d}-E_{r, c}}{\left\|x_{d}-x_{c}\right\|} \\
+\sum_{d \in \mathcal{N}(c)} \frac{a c L_{d c}}{3 \sigma_{R, d c}} \frac{T_{r, d}^{4}-T_{r, c}^{4}}{\left\|x_{d}-x_{c}\right\|} \frac{T_{r, d}-T_{r, c}}{T_{r, c}\left(T_{r, d}+T_{r, c}\right)} \\
+\frac{a c \sigma_{P, c} v_{c}}{T_{r, c} T_{e, c}}\left(T_{e, c}-T_{r, c}\right)\left(T_{e, c}^{4}-T_{r, c}^{4}\right)+v_{c} \mathcal{K}_{c} \frac{\left(T_{e, c}-T_{i, c}\right)^{2}}{T_{e, c} T_{i, c}}
\end{array}\right] .
\end{aligned}
$$

Integrating over the space domain finally leads to the expected result.

Property A.2 (Discrete total energy conservation). The total discrete energy is conserved

$$
\sum_{c} m_{c}\left(\frac{\phi_{r, c}^{n+1}}{\rho_{c}^{n+1}}+\varepsilon_{e, c}^{n+1}+\varepsilon_{i, c}^{n+1}+\frac{\left\|\boldsymbol{u}_{c}^{n+1}\right\|^{2}}{2}\right)=\sum_{c} m_{c}\left(\frac{\phi_{r, c}^{n}}{\rho_{c}^{n}}+\varepsilon_{e, c}^{n}+\varepsilon_{i, c}^{n}+\frac{\left\|\boldsymbol{u}_{c}^{n}\right\|^{2}}{2}\right) .
$$

Proof. Multiplying the second equation of system (2.12) by $\left(\boldsymbol{u}_{c}^{n+1}+\boldsymbol{u}_{c}^{n}\right) / 2$, one obtains

$$
\frac{m_{c}}{2 \Delta t}\left(\left\|\boldsymbol{u}_{c}^{n+1}\right\|^{2}-\left\|\boldsymbol{u}_{c}^{n}\right\|^{2}\right)=\sum_{p \in \mathcal{P}(c)} f_{p c}^{n} \cdot\left(\frac{\boldsymbol{u}_{c}^{n+1}+\boldsymbol{u}_{c}^{n}}{2}\right) .
$$

On the other hand, summing the equations of system (2.31), one gets

$$
\begin{aligned}
& \frac{m_{c}}{\Delta t}\left(\frac{\phi_{r, c}^{n+1, k+1}}{\rho_{c}^{n+1}}-\frac{\phi_{r, c}^{n}}{\rho_{c}^{n}}+\frac{\phi_{e, c}^{n+1, k+1}-\phi_{e, c}^{n}}{\beta_{e, c}^{n+1, k}}+\frac{\phi_{i, c}^{n+1, k+1}-\phi_{i, c}^{n}}{\beta_{i, c}^{n+1, k}}\right) \\
= & \sum_{p \in \mathcal{P}(c)} f_{p c}^{n} \cdot\left(\boldsymbol{u}_{p}^{n}-\frac{\boldsymbol{u}_{c}^{n+1}+\boldsymbol{u}_{c}^{n}}{2}\right)+\sum_{d \in \mathcal{N}(c)} \frac{c L_{d c}}{3 \sigma_{R, d c}^{n+1, k}} \frac{\phi_{r, d}^{n+1, k+1}-\phi_{r, c}^{n+1, k+1}}{\left\|x_{c}-x_{d}\right\|_{2}} .
\end{aligned}
$$


Now, by using the definition of $\beta_{\alpha}$, we have

$$
\begin{aligned}
& \frac{m_{c}}{\Delta t}\left(\frac{\phi_{r, c}^{n+1, k+1}}{\rho_{c}^{n+1}}-\frac{\phi_{r, c}^{n}}{\rho_{c}^{n}}+\frac{\varepsilon_{e, c}^{n+1, k}-\varepsilon_{e, c}^{n}}{\phi_{e, c}^{n+1, k}-\phi_{e, c}^{n}}\left(\phi_{e, c}^{n+1, k+1}-\phi_{e, c}^{n}\right)+\frac{\varepsilon_{i, c}^{n+1, k}-\varepsilon_{i, c}^{n}}{\phi_{i, c}^{n+1, k}-\phi_{i, c}^{n}}\left(\phi_{i, c}^{n+1, k+1}-\phi_{i, c}^{n}\right)\right) \\
= & \sum_{p \in \mathcal{P}(c)} f_{p c}^{n} \cdot\left(\boldsymbol{u}_{p}^{n}-\frac{\boldsymbol{u}_{c}^{n+1}+\boldsymbol{u}_{c}^{n}}{2}\right)+\sum_{d \in \mathcal{N}(c)} \frac{c L_{d c}}{3 \sigma_{R, d c}^{n+1, k}} \frac{\phi_{r, d}^{n+1, k+1}-\phi_{r, c}^{n+1, k+1}}{\left\|x_{c}-x_{d}\right\|_{2}} .
\end{aligned}
$$

At convergence of the iterative procedure $(k \rightarrow+\infty)$, the previous equation reads

$$
\begin{aligned}
& \frac{m_{c}}{\Delta t}\left(\frac{\phi_{r, c}^{n+1}}{\rho_{c}^{n+1}}-\frac{\phi_{r, c}^{n}}{\rho_{c}^{n}}+\varepsilon_{e, c}^{n+1}-\varepsilon_{e, c}^{n}+\varepsilon_{i, c}^{n+1}-\varepsilon_{i, c}^{n}\right) \\
= & \sum_{p \in \mathcal{P}(c)} f_{p c}^{n} \cdot\left(\boldsymbol{u}_{p}^{n}-\frac{\boldsymbol{u}_{c}^{n+1}+\boldsymbol{u}_{c}^{n}}{2}\right)+\sum_{d \in \mathcal{N}(c)} \frac{c L_{d c}}{3 \sigma_{R, d c}^{n+1}} \frac{\phi_{r, d}^{n+1}-\phi_{r, c}^{n+1}}{\left\|x_{c}-x_{d}\right\|_{2}} .
\end{aligned}
$$

By adding Eqs. (A.5) and (A.7) we get

$$
\begin{aligned}
& \frac{m_{c}}{\Delta t}\left(\frac{\phi_{r, c}^{n+1}}{\rho_{c}^{n+1}}-\frac{\phi_{r, c}^{n}}{\rho_{c}^{n}}+\varepsilon_{e, c}^{n+1}-\varepsilon_{e, c}^{n}+\varepsilon_{i, c}^{n+1}-\varepsilon_{i, c}^{n}+\frac{\left\|\boldsymbol{u}_{c}^{n+1}\right\|^{2}}{2}-\frac{\left\|\boldsymbol{u}_{c}^{n}\right\|^{2}}{2}\right) \\
= & \sum_{p \in \mathcal{P}(c)} f_{p c}^{n} \cdot \boldsymbol{u}_{p}^{n}+\sum_{d \in \mathcal{N}(c)} \frac{c L_{d c}}{3 \sigma_{R, d c}^{n+1}} \frac{\phi_{r, d}^{n+1}-\phi_{r, c}^{n+1}}{\left\|x_{c}-x_{d}\right\|_{2}} .
\end{aligned}
$$

Since all terms of the right-hand side of the previous equation are conservative the property is proven.

\section{References}

[1] X. Blanc and E. Labourasse. A positive scheme for diffusion problems on deformed meshes. Z. Angew. Math. Mech., 96:660-680, 2015.

[2] J. Breil. Numerical Methods for Lagrangian and Arbitrary-Lagrangian-Eulerian Hydrodynamic Contribution to the Simulation of High-Energy-Density-Physics Problems, Université de Bordeaux, HDR, 2017.

[3] J.S. Camier and F. Hermeline. A monotone nonlinear finite volume method for approximating diffusion operators on general meshes. Applied Numerical Mathematics, 107, 6:496-519, 2016.

[4] J.I. Castor. Radiation Hydrodynamics, Cambridge University Press. 2004.

[5] E. Chatzopoulos and K. Weide. Gray Radiation Hydrodynamics with the Flash Code for Astrophysical Applications. The American Astronomical Society, 2019.

[6] J. Cheng, C.W. Shu, and Q. Zeng. A conservative lagrangian scheme for solving compressible fluid flows with multiple internal energy equations. Communications in Computational Physics, 12(5):13071328, 2012.

[7] A. Decoster. Fluids equations and transport coefficients of plasmas. Modeling of Collisions, Series in Applied Mathematics, 1998. 
[8] C. Enaux, S. Guisset, C. Lasuen, and Q. Ragueneau. Numerical resolution of a three temperature plasma model. Journal of Scientific Computing, Springer, 2020.

[9] T.M. Evans and J.D. Densmore. Methods for coupling radiation, ion and electron energies in grey implicit Monte Carlo. J. Comput. Phys., 225:1695-1720, 2007.

[10] M. Fatenejad, B. Fryxell, J. Wohlbier, E. Myra, D. Lamb, C. Fryer, and C. Graziani. Collaborative comparison of simulation codes for high-energy-density physics applications. High Energy Density Physics, 9:63-66, 2013.

[11] T. Feng, H. An, X. Yu, Q. Li, and R. Zhang. On linearization and preconditioning for radiation diffusion coupled to material thermal conduction equations. J. Comput. Phys., 236:2840, 2013.

[12] J.A. Fleck and J.D. Cummings. An implicit Monte Carlo scheme for calculating time and frequency dependent nonlinear radiation transport. J. Comput. Phys., 8:313-342, 1971.

[13] F. Graziani. Radiation Diffusion: An Overview of Physical and Numerical Concepts, pages 29-66, 2005.

[14] C. W. Hirt, A. A. Amsden, and J. L. Cook. An arbitrary Lagrangian-Eulerian computingmethod for all flow speeds. J. Comput. Phys., 14:277-253, 1974.

[15] R. Kamm and F.X. Timmes. On efficient generation of numerically robust Sedov solutions. LANL Report LA-UR-07-2849, 2007.

[16] D.A. Knoll, R.B. Lowrie, and J.E. Morel. Numerical analysis of time integration errors for nonequilibrium radiation diffusion. J. Comput. Phys., 226:1332-1347, 2007.

[17] E. W. Larsen and B. Mercier. Analysis of a Monte Carlo method for nonlinear radiative transfer. Journal of Computational Physics, 71(1):50-64, 1987.

[18] R.B. Lowrie and J.D. Edwards. Shock wave solutions for radiation hydrodynamics. Joint International Topical Meeting on Math and Computations and Supercomputing in Nuclear Applications, Monterey, CA, 2007.

[19] P.H. Maire. Contribution to the numerical modeling of inertial confinement fusion. Habilitation à Diriger des Recherches, 2011.

[20] P.H. Maire, R. Abgrall, J. Breil, and J. Ovadia. A cell-centered lagrangian scheme for twodimensional compressible flow problems. SIAM J.on Scientific Computing, 29:1781-1824, 2007.

[21] A. Marboeuf. Schémas ALE multi-matériaux totalement conservatifs pour l'hydrodynamique. PhD thesis, École Ploytechnique, 2018.

[22] D. Mihalas and B. Mihalas. Foundations of Radiation Hydrodynamics. Oxford University Press, 1984.

[23] D. Mihalas and B. Mihalas. Foundations of Radiation Hydrodynamics. Oxford University Press Oxford, 1964.

[24] V.A. Mousseau and D.A. Knoll. New physical-based preconditioning of implicit methods for non-equilibrium radiation diffusion. J. Comput. Phys., 190:42-51, 2003.

[25] G. Olson, L. Auer, and M. Hall. Diffusion, P1, and other approximate forms of radiation transport. Journal of Quantitative Spectroscopy and Radiative Transfer, 64:619-634, 2000.

[26] G. Peng, Z. Gao, and X. Feng. A stabilized extremum preserving scheme for nonlinear parabolic equation on polygonal meshes. International Journal for Numerical Methods in Fluids, 90:340-356, 2019.

[27] G. Peng, Z. Gao, W. Yan, and X. Feng. A positivity-preserving finite volume scheme for three-temperature radiation diffusion equations. Applied Numerical Mathematics, 152:125$140,2020$.

[28] W.J. Rider and D.B. Kothe. Reconstructing volume tracking. Journal of Computational 
Physics, 141(2):112-152, 1998.

[29] C.D. Sijoy and S. Chaturvedi. Combining node-centered parallel radiation transport and higher-order multi-material cell-centered hydrodynamics methods in three-temperature radiation hydrodynam- ics code TRHD. Computer Physics Communications, 203:94-109, 2016.

[30] C.D. Sijoy and S. Chaturvedi. TRHD: Three-temeprature radiation-hydrodynamics code with an implicit non-equilibrium radiation transport using a cell-centered monotonic finite volume scheme on unstructured-grids. Computer Physics Communications, 190:98-119, 2015.

[31] L. Spitzer and R. Härm. Transport phenomena in a completely ionized gas. Phys. Rev., 89:977-981, 1953.

[32] T.J. Urbatsch and T.M Evans. Milagro version 2. An implicit monte carlo code for thermal radiative transfer: Capabilities, development and usage. LA-14195-MS, 2006.

[33] Y. Yu, X. Chen, and G. Yuan. A finite volume scheme preserving maximum principle for the system of radiation diffusion equations with three-temperature. SIAM Journal on Scientific Computing, 41(1):B93-B113, 2019.

[34] B. Zeldovich and P. Raizer. The Mechanics and Thermodynamics of Continua. Physics of Shock Waves and High-Temperature Hydrodynamic Phenomena, volume 2. Academic Press, 1966.

[35] W. Zhang, L. Howell, A. Almgren, A. Burrows, and J. Bell. CASTRO: A new compressible astrophysical solver. II. Gray radiation hydrodynamics. The Astrophysical Journal Supplement Series, 196:20 (16pp), 2011. 